TRANSACTIONS OF THE

AMERICAN MATHEMATICAL SOCIETY

Volume 353, Number 7, Pages 2915-2939

S 0002-9947(01)02687-3

Article electronically published on March 8, 2001

\title{
MINIMAL PROJECTIVE RESOLUTIONS
}

\author{
E. L. GREEN, Ø. SOLBERG, AND D. ZACHARIA
}

Dedicated to Helmut Lenzing for his 60th birthday

\begin{abstract}
In this paper, we present an algorithmic method for computing a projective resolution of a module over an algebra over a field. If the algebra is finite dimensional, and the module is finitely generated, we have a computational way of obtaining a minimal projective resolution, maps included. This resolution turns out to be a graded resolution if our algebra and module are graded. We apply this resolution to the study of the Ext-algebra of the algebra; namely, we present a new method for computing Yoneda products using the constructions of the resolutions. We also use our resolution to prove a case of the "no loop" conjecture.
\end{abstract}

\section{INTRODUCTION}

In the study of homological properties of rings and modules, projective resolutions are a basic tool. Such resolutions occur naturally in commutative ring theory, the representation theory of finite dimensional algebras, group representation theory, algebraic geometry, and algebraic topology [A, AG, F, [H1, HZ]. On the other hand, with the introduction of computers, computational and algorithmic techniques have grown in importance [Ba FGKK]. Both theoretical and practical results are needed. This paper presents a new method of constructing projective resolutions in a broad setting which has both theoretical and computational implications. In particular, in the graded and finite dimensional cases, our results provide a recursive procedure for computing minimal projective resolutions.

The class of algebras studied in this paper consists of quotients of path algebras. We fix a field $K$ for the remainder of this paper. If $Q$ is a finite directed graph, which we call a quiver, then the path algebra, $K Q$, is the $K$-algebra with $K$-basis consisting of finite directed paths in $Q$. Thus, elements of $K Q$ consist of $K$-linear combinations of paths in $Q$. The multiplicative structure on basis elements $p$ and $q$ is defined by concatenation $p q$ if the terminus of $p$ equals the origin of $q$, and by 0 otherwise. We view the vertices as paths of length 0 with multiplication given as follows. If $v$ and $w$ are vertices and $p$ is a path, we let $v \cdot w$ be $v$ if $v=w$ and 0 otherwise. We let $v \cdot p=p$ if $v$ is the origin of $p$ and 0 otherwise, and we define $p \cdot w$ similarly. The multiplication on paths is extended linearly to arbitrary elements of $K Q$. Note that the free associative $K$-algebra on $n$ noncommuting variables is

Received by the editors September 21, 1998 and, in revised form, January 3, 2000.

2000 Mathematics Subject Classification. Primary 16E05, 18G10; Secondary 16P10.

Key words and phrases. Projective resolutions, finite dimensional and graded algebras.

Partially supported by a grant from the NSA.

Partially supported by NRF, the Norwegian Research Council.

(C)2001 American Mathematical Society 
isomorphic to the path algebra $K Q$ where $Q$ has one vertex and $n$ loops. We let $Q_{0}$ denote the vertex set of $Q$.

Let $Q$ be a quiver and $I$ be a (two-sided) ideal in the path algebra $K Q$. Let $\Lambda$ denote $K Q / I$ for the remainder of the introduction. The algebras in this class include all affine (that is, finitely generated) associative $K$-algebras. Every finite dimensional $K$-algebra is Morita equivalent to an algebra in this class if $K$ is algebraically closed. Furthermore, this class includes graded $K$-algebras $\Lambda=\Lambda_{0} \oplus \Lambda_{1} \oplus \Lambda_{2} \oplus \cdots$ where $\Lambda_{0}$ is a product of a finite number of copies of $K$, each $\Lambda_{i}$ is a finite dimensional $K$-vector space and $\Lambda$ is generated in degrees 0 and 1 ; that is, for $i, j \geq 0$, $\Lambda_{i} \Lambda_{j}=\Lambda_{i+j}$.

Let $M$ be a $\Lambda=K Q / I$-module. Let $F \rightarrow M \rightarrow 0$ be an exact sequence of $K Q$ modules with $F=\coprod_{v \in Q_{0}} v K Q$. A main theme of the paper is the construction of a filtration of $F$ by $K Q$-submodules which contains all the information needed to construct the $\Lambda$-projective resolution of $M$, the $\Lambda$-syzygies and the Yoneda product of extensions of $\Lambda$-modules. In particular, we find a filtration

$$
\cdots \subset F^{n} \subset F^{n-1} \subset \cdots \subset F^{1} \subset F^{0},
$$

such that $F=F^{0}, M=F^{0} / F^{1}$ and

$$
\cdots F^{n} / F^{n} I \rightarrow F^{n-1} / F^{n-1} I \rightarrow \cdots \rightarrow F^{1} / F^{1} I \rightarrow F^{0} / F^{0} I \rightarrow M \rightarrow 0
$$

is a $\Lambda$-projective resolution of $M$ with the maps induced by the inclusions of the filtration. For the basic construction, we do not assume that $\Lambda$ is finite dimensional, or even noetherian. Furthermore, we do not assume that the $\Lambda$-module $M$ is finitely generated. For our minimality results, $M$ will be finitely generated and $\Lambda$ either finite dimensional or graded.

We provide a recursive formula to compute $F^{n}$ as a $K Q$-submodule of $F^{n-1}$ from the previously obtained $F^{n-1} \subset F^{n-2}$. To explicitly find $F^{n}$ from our formula, one must write an intersection of certain submodules of a projective $K Q$-module as a direct sum of cyclic submodules. A method for finding the generators of these cyclic submodules employs the theory of right Gröbner bases, and will appear elsewhere.

Our construction resembles earlier resolutions of Bongartz, Butler, Eilenberg, Eilenberg-Nagao-Nakayama and Gruenberg [Bo, E, ENN]. Their resolutions are almost never minimal in the finite dimensional case, and deal only with resolutions of semisimple modules. We recall their resolution. Let $J$ denote the ideal of $K Q$ generated by the arrows of $Q$. Furthermore, assume that $J^{N} \subseteq I \subseteq J^{2}$ for some positive integer $N \geq 2$. Then we have the filtration

$$
\cdots \subset J I^{n} \subset I^{n} \subset \cdots \subset I^{3} \subset J I^{2} \subset I^{2} \subset J I \subset I \subset J \subset K Q .
$$

Note that $J / I$ is the Jacobson radical of $\Lambda$ and that $\Lambda /(J / I)$ is isomorphic to $K Q / J$. One gets the following $\Lambda$-projective resolution of $K Q / J$

$$
\cdots \rightarrow I^{n} / I^{n+1} \rightarrow J I^{n-1} / J I^{n} \rightarrow \cdots \rightarrow I / I^{2} \rightarrow J / J I \rightarrow K Q / I \rightarrow K Q / J \rightarrow 0,
$$

where the maps are induced by the inclusions.

The paper is organized as follows. In the first section, we give a general construction of a projective resolution of an arbitrary $\Lambda$-module $M$, where $\Lambda$ is a quotient of a path algebra. We show that if $\Lambda$ is a right noetherian algebra and $M$ is a finitely generated $\Lambda$-module, then the resolution is finitely generated. If $\Lambda$ is graded and $M$ is a graded module, we show how to modify the construction to obtain a graded projective resolution. 
In the second section, we provide algorithmic techniques to adjust the construction to obtain minimal projective resolutions in both the finite dimensional and the graded cases. The section ends with explicit computations of syzygies and Ext-groups.

Section 3 deals with Ext-algebras. If $\Lambda_{0}$ denotes $\Lambda$ modulo its radical in the finite dimensional case, or, $\Lambda$ modulo its graded radical in the graded case, then we study the algebraic structure of

$$
E(\Lambda)=\coprod_{n \geq 0} \operatorname{Ext}_{\Lambda}^{n}\left(\Lambda_{0}, \Lambda_{0}\right)
$$

Furthermore, if $M$ is either a finite dimensional $\Lambda$-module or a graded $\Lambda$-module, we investigate the $E(\Lambda)$-module structure of $E(M)=\bigsqcup_{n \geq 0}\left(M, \Lambda_{0}\right)$. A major result of the paper is that this module structure is included in the information obtained in the construction of the resolution. In particular, one need not "lift maps" to find the Yoneda products.

We apply our techniques to prove one case of the No Loop Conjecture in section four. Namely, we prove that if $\Lambda$ is a finite dimensional $K$-algebra and $a$ is a loop at the vertex $v$ such that $a^{n}$ is the first power of $a$ belonging to the ideal $I$ but $a^{n}$ is not in $J I+I J$, then $\operatorname{Ext}_{\Lambda}^{n}(S, S) \neq(0)$, for all $n \geq 1$, where $S$ is the simple $\Lambda$-module corresponding to the vertex $v$.

In the final section we investigate the influence of the characteristic of the ground field $K$ on the structure of projective resolutions. Other than some new examples, we show that if the global dimension of $\Lambda$ is bounded by 2 in one characteristic, then the global dimension will be finite in all characteristics. We also provide an example of an algebra that has infinite global dimension in only one characteristic.

Finally, we note that all modules will be right modules unless otherwise stated. We also introduce some terminology. We say that an element $x$ in the path algebra $K Q$ is right uniform, if $x \neq 0$ and there is a vertex $v$ such that $x v=x$. Note that if $x \neq 0$ is an element of $K Q$, then $x=\sum_{v \in Q_{0}} x v$. Hence, every nonzero element of $K Q$ is a sum of right uniform elements. From a different point of view, $K Q=\coprod_{v \in Q_{0}} K Q v$ as left modules. Hence, every nonzero element is a sum of right uniform elements in a unique way. An element is right uniform if and only if it is nonzero and a linear combination of paths ending at a single vertex. Finally, note that if $x$ is a right uniform element with $x v=x$ for some $v \in Q_{0}$, then $x K Q$ is a right projective $K Q$-module isomorphic to $v K Q$.

Acknowledgment. The major work on this paper was done when the last two authors visited the Department of Mathematics at Virginia Tech. We would like to thank the first author and the department for their hospitality and effort in making our stay there a very pleasant and interesting one. The authors also thank M. C. R. Butler and the referee for their comments and suggestions, which are addressed in an appendix to the paper.

\section{The Resolution}

Let $Q$ be a finite quiver, and let $R=K Q$ denote the path algebra of $Q$ over a field $K$. Let $I$ be a two-sided ideal in $R$ such that $I \subseteq J^{2}$, where $J$ denotes the ideal of $R$ generated by the arrows of the quiver $Q$. Let $\Lambda=R / I$ be the quotient algebra, and let $M$ be a right $\Lambda$-module. In this section we construct, in an algorithmic way, a projective resolution $(\mathcal{P}, \delta)$ of $M$ over $\Lambda$. This resolution need not be finitely 
generated in general, but it is when $\Lambda$ is noetherian and $I$ is finitely generated as a right ideal in $R$. In particular, if $I$ is an admissible ideal of $R$, that is, $J^{N} \subseteq I \subseteq J^{2}$ for some $N>1$, then $\Lambda$ is a finite dimensional $K$-algebra and the resolution $(\mathcal{P}, \delta)$ becomes a finitely generated resolution. In the next section we also show how, in this case, we can adjust $\mathcal{P}$ in an algorithmic way to obtain a minimal projective resolution of $M_{\Lambda}$. Moreover, if $\Lambda$ is graded by the natural grading induced from the length grading on $R$, then the resolution constructed for a graded $\Lambda$-module is also graded.

We shall use the following well-known properties of the path algebra $R=K Q$ : (a) for every $x$ in $R$, the $R$-module $x R$ is projective, and, (b) for each $R$-submodule $Y$ of $\amalg_{i} x_{i} R$ with $x_{i}$ in $R$, we have $Y=\amalg_{j} y_{j} R$ for some $y_{j}$ in $\amalg_{i=1}^{k} x_{i} R$ (of course, if $Y$ is finitely generated, then we can write $Y=\amalg_{j=1}^{t} y_{j} R$ for some finite set $\left\{y_{1}, \ldots, y_{t}\right\}$ in $\left.\left.\amalg_{i} x_{i} R, \mathrm{G}\right]\right)$. We now introduce the notation that will be needed in defining the resolution $(\mathcal{P}, \delta)$ of $M$, and, throughout this paper.

Choose a family $\left\{f_{i}^{0}\right\}_{i \in A}$ of elements of $R$ such that the projective $\Lambda$-module $\amalg_{i \in A} f_{i}^{0} R / \amalg_{i \in A} f_{i}^{0} I$ maps onto $M$. Without loss of generality we choose the family to consist of vertices in $R$ (repetitions allowed). We have

$$
0 \rightarrow \Omega_{R}^{1}(M) \rightarrow \amalg_{i \in A} f_{i}^{0} R \rightarrow M \rightarrow 0,
$$

and, we then choose a set $\left\{f_{i}^{1 *}\right\}$ of elements of $\amalg_{i \in A} f_{i}^{0} R$ such that $\Omega_{R}^{1}(M)=$ $\amalg_{i} f_{i}^{1 *} R$. Discard all the elements $f_{i}^{1 *}$ that are in $\amalg_{i \in A} f_{i}^{0} I$ and denote by $\left\{f_{i}^{1}\right\}$ those $f^{1 *}$ 's that are not elements of $\amalg_{i \in A} f_{i}^{0} I$. Assume that we have constructed families of elements of $\amalg_{i \in A} f_{i}^{0} R$ : $\left\{f_{i}^{k}\right\}_{i}$ for each $k=0, \ldots, n$. We now construct the family $\left\{f_{i}^{n+1}\right\}_{i}$ as follows. We consider the intersection $\left(\amalg_{i} f_{i}^{n} R\right) \cap\left(\amalg_{j} f_{j}^{n-1} I\right)$. We stop if the intersection is zero, and we set it equal to some $\amalg_{l} f_{l}^{n+1 *} R$ otherwise. Discard all the elements of the form $f^{n+1 *}$ that are in $\amalg_{i} f_{i}^{n} I$, and denote the remaining ones by $\left\{f_{i}^{n+1}\right\}_{i}$. If each element of the form $f^{n+1 *}$ is in $\amalg_{i} f_{i}^{n} I$, we again stop at this stage of the construction. Note that we may assume that for each $n$, each element $f_{i}^{n}$ can be chosen to be right uniform, that is, there is a vertex $v$ (dependent on $f_{i}^{n}$ ) such that $f_{i}^{n} v=f_{i}^{n}$. An element of $R$ is uniform if it is a linear combination of paths in $R$, all starting at one vertex, and, all ending at one vertex. We also note that, for each $n>0$, we have a representation of $f_{k}^{n}$ in $\amalg f_{i}^{n-1} R$ as follows:

$$
f_{k}^{n}=\sum_{i} f_{i}^{n-1} h_{i, k}^{n-1, n}
$$

for scalars $h_{i, k}^{n-1, n}$ in $R$. Note that for each $k$, all but a finite number of $h_{i, k}^{n-1, n}$ are zero. It is convenient to encode this information in the matrix $\left(h_{i, k}^{n-1, n}\right)$. Furthermore, since the $f_{k}^{n}$ 's and the $f_{i}^{n-1}$ 's are right uniform, it follows that each $h_{i, k}^{n-1, n}$ is uniform.

Setting $F^{n}=\amalg_{i} f_{i}^{n} R$, from our construction, we have the following filtration of the right projective $R$-module $F^{0}$ :

$$
\cdots \subseteq F^{n} \subseteq F^{n-1} \subseteq \cdots \subseteq F^{2} \subseteq F^{1} \subseteq F^{0} .
$$

Definition 1.1. For each $n \geq 0$ let $P_{n}=\amalg_{i} f_{i}^{n} R / \amalg_{i} f_{i}^{n} I$, and let $\delta^{n}: P_{n} \rightarrow P_{n-1}$ be the homomorphism induced by the inclusion $\amalg f_{i}^{n} R \subset \amalg f_{j}^{n-1} R$. We also define the matrix $\left(\bar{h}_{i, k}^{n-1, n}\right)$ where $\bar{h}$ denotes the image in $\Lambda$ of the element $h$ in $R$. 
Note that the boundary maps $\delta^{n}$ are, in fact, determined by multiplication by the matrix $\left(\bar{h}^{n-1, n}\right)$, which gives a formula for the coordinates.

We can now state our first result.

Theorem 1.2. $(\mathcal{P}, \delta): \cdots \rightarrow P_{n} \stackrel{\delta^{n}}{\longrightarrow} P_{n-1} \rightarrow \cdots \rightarrow P_{1} \stackrel{\delta^{1}}{\longrightarrow} P_{0} \rightarrow M \rightarrow 0$ is a projective resolution of $M$ over $\Lambda$.

Proof. It is clear that for each $n \geq 0$, the modules $P_{n}$ are projective $\Lambda$-modules. From the following commutative diagram with exact rows

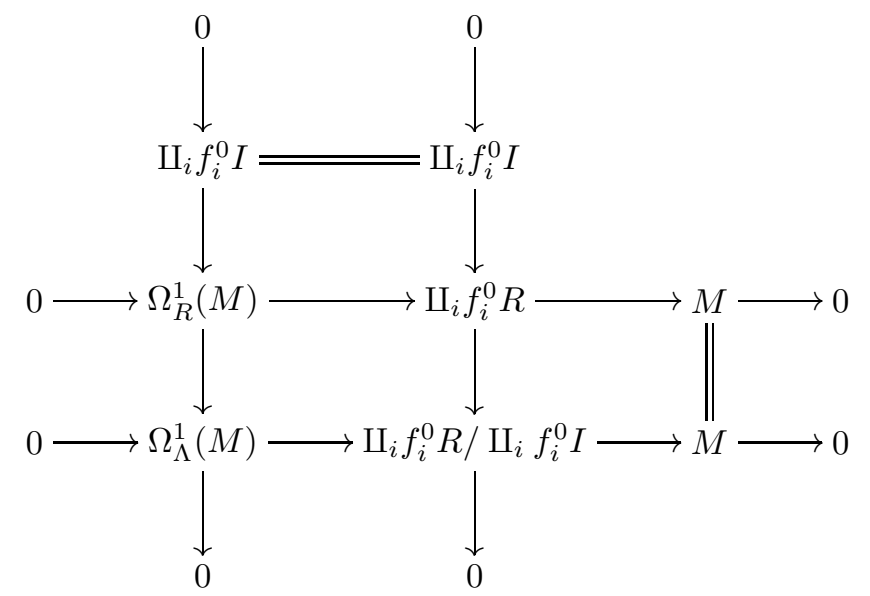

it follows that we have exactness at $P_{0}$. We now show that for each $n>0$, we have $\delta^{n} \delta^{n+1}=0$. We must show that $\left(\bar{h}_{i, k}^{n-1, n}\right)\left(\bar{h}_{k, l}^{n, n+1}\right)$ is the zero matrix, or, equivalently that for each $i$ and $l$, the sum $\sum_{k} h_{i, k}^{n-1, n} h_{k, l}^{n, n+1}$ is in $I$. But $\sum_{i} f_{i}^{n-1}\left(\sum_{k} h_{i, k}^{n-1, n} h_{k, l}^{n, n+1}\right)$ is an element of $\amalg f_{i}^{n-1} R$, and we also have

$$
\begin{aligned}
\sum_{i} f_{i}^{n-1}\left(\sum_{k} h_{i, k}^{n-1, n} h_{k, l}^{n, n+1}\right) & =\sum_{k}\left(\sum_{i} f_{i}^{n-1} h_{i, k}^{n-1, n}\right) h_{k, l}^{n, n+1} \\
& =\sum_{k} f_{k}^{n} h_{k, l}^{n, n+1}=f_{l}^{n+1}
\end{aligned}
$$

which lies in $\amalg f_{i}^{n-1} I$. We infer from the uniqueness of the representations as elements of direct sums that for each $i$ and $l$, the element $\sum_{k} h_{i, k}^{n-1, n} h_{k, l}^{n, n+1}$ is in $I$.

It remains to show that for each $n, \operatorname{Ker} \delta^{n} \subseteq \operatorname{Im} \delta^{n+1}$. Let $\left(\bar{x}_{k}\right)_{k}$ be in the kernel of $\delta^{n}$. Therefore, $\sum_{k} \bar{h}_{i, k}^{n-1, n} \bar{x}_{k}=\overline{0}$ for all $i$, or, equivalently $\sum_{k} h_{i, k}^{n-1, n} x_{k}$ is in the ideal $I$ for all $i$. On the other hand,

$$
\sum_{i} f_{i}^{n-1}\left(\sum_{k} h_{i, k}^{n-1, n} x_{k}\right)=\sum_{k}\left(\sum_{i} f_{i}^{n-1} h_{i, k}^{n-1, n}\right) x_{k}=\sum_{k} f_{k}^{n} x_{k}
$$

is an element of $\amalg_{k} f_{k}^{n} R$ and, since $\sum_{i} f_{i}^{n-1}\left(\sum_{k} h_{i, k}^{n-1, n} x_{k}\right)$ is also in $\amalg_{i} f_{i}^{n-1} I$, we have that the element $\sum_{i} f_{i}^{n-1}\left(\sum_{k} h_{i, k}^{n-1, n} x_{k}\right)$ is in $\amalg f_{j}^{n+1 *} R$. Therefore, we can rewrite it as

$$
\sum_{i} f_{i}^{n-1}\left(\sum_{k} h_{i, k}^{n-1, n} x_{k}\right)=\sum_{j} f_{j}^{n+1} \gamma_{j}+u
$$


where $\gamma_{j}$ is an element in $R$ and $u$ is an element of $\amalg f_{k}^{n} I$. We claim that we have $\delta^{n+1}\left(\left(\bar{\gamma}_{j}\right)_{j}\right)=\left(\bar{x}_{k}\right)_{k}$, where $\bar{\gamma}_{j}$ denotes the image in $\Lambda$ of the element $\gamma_{j}$ in $R$. To prove this we have

$$
\delta^{n+1}\left(\left(\bar{\gamma}_{j}\right)_{j}\right)=\left(\bar{h}_{k, j}^{n, n+1}\right)\left(\bar{\gamma}_{j}\right)_{j}=\left(\sum_{j} \bar{h}_{k, j}^{n, n+1} \bar{\gamma}_{j}\right)_{k}
$$

But, we have

$$
\sum_{k} f_{k}^{n}\left(\sum_{j} h_{k, j}^{n, n+1} \gamma_{j}\right)=\sum_{j} f_{j}^{n+1} \gamma_{j}=\sum_{k} f_{k}^{n} x_{k}
$$

modulo $\amalg f_{k}^{n} I$. Hence, we infer that for each $k$ we get $\sum_{j} h_{k, j}^{n, n+1} \gamma_{j}=x_{k}$ modulo $I$. This proves that $\operatorname{Ker} \delta^{n} \subseteq \operatorname{Im} \delta^{n+1}$ and the proof is complete.

We show next that the resolution constructed above is a finitely generated resolution if we assume in addition that $\Lambda$ is noetherian and $I$ is finitely generated as a right ideal in $R$.

Theorem 1.3. Assume that $\Lambda$ is noetherian and that $I$ is finitely generated as a right ideal of $R=K Q$. Let $M_{\Lambda}$ be finitely generated. Then the resolution $(\mathcal{P}, \delta)$ of $M_{\Lambda}$ is finitely generated.

Proof. First observe that we may choose $f_{1}^{0}, \ldots, f_{k}^{0}$ in $R$ such that $\amalg_{i=1}^{k} f_{i}^{0} R / \amalg_{i=1}^{k}$ $f_{i}^{0} I$ maps onto $M$. To prove the theorem, it is enough to show that, for each $n>0$, the direct sums $\amalg_{i} f_{i}^{n *} R$ are finite. We prove this first for $n=1$. We have the exact sequence of $R$-modules,

$$
0 \rightarrow \amalg_{i=1}^{k} f_{i}^{0} I \rightarrow \Omega_{R}^{1}(M) \rightarrow \Omega_{\Lambda}^{1}(M) \rightarrow 0,
$$

and, since both ends are finitely generated, then so is the middle term. But $\Omega_{R}^{1}(M)=\amalg_{i} f_{i}^{1 *} R$ hence this sum must be finite. We show now by induction, that, for each $n>1$ we have $\Omega_{R}^{1}\left(\Omega_{\Lambda}^{n-1}(M)\right)=\amalg_{i} f_{i}^{n *} R$ and that they are all finitely generated. (Here by $\Omega_{\Lambda}^{k}(M)$ we mean the kernel of the map $\amalg_{i} f_{i}^{k-1} R / \amalg_{i} f_{i}^{k-1} I \rightarrow$ $\Omega_{\Lambda}^{k-1}(M)$.) If $n \geq 2$, we have the following exact commutative diagram:

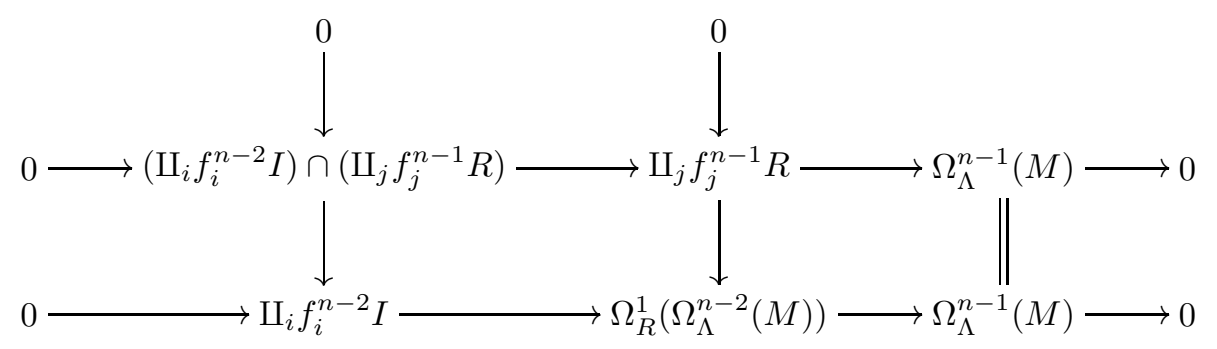

which shows that $\Omega_{R}^{1}\left(\Omega_{\Lambda}^{n-1}(M)\right)=\amalg_{t} f_{t}^{n *} R$, and the diagram 


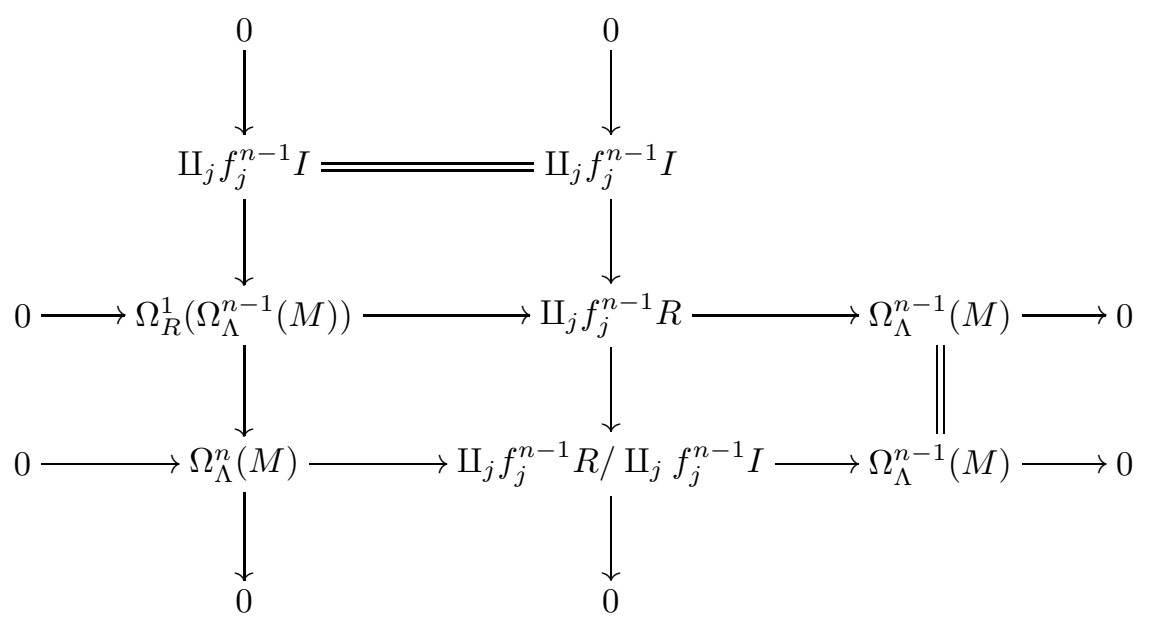

and, from the left vertical exact sequence, an easy induction argument shows that $\Omega_{R}^{1}\left(\Omega_{\Lambda}^{n-1}(M)\right)$ is finitely generated. Thus the sum $\amalg_{t} f_{t}^{n *} R$ is finite. The proof of the theorem is now complete.

Remark 1.4. If $I$ is an admissible ideal in $R$, then $I$ is finitely generated as a right ideal in $R$ and $\Lambda=R / I$ is a finite dimensional $K$-algebra, hence noetherian. Therefore, as a corollary of the above the resolution is finitely generated for any finitely generated $\Lambda$-module $M_{\Lambda}$ when $I$ is an admissible ideal.

The path algebra $R=K Q$ has a natural grading $R=\amalg_{i}(K Q)_{i}$ where, for each $i,(K Q)_{i}$ denotes the $K$-vector space spanned by the paths of $Q$ of length $i$. Each $(K Q)_{i}$ is endowed with an obvious $(K Q)_{0^{-}}(K Q)_{0}$-bimodule structure, and, $J=\amalg_{i \geq 1}(K Q)_{i}$ is the graded radical of $R$. If $I \subseteq J^{2}$ is a two-sided ideal generated by homogeneous elements, then $\Lambda=K Q / I$ has an induced grading. In this case we say that $\Lambda$ is length graded. By a graded $\Lambda$-module $M$, we will always mean a graded $\Lambda$-module $M=\amalg_{i \in \mathbb{Z}} M_{i}$ such that, $M_{i}=(0)$ for sufficiently small $i$, and, each $M_{i}$ is a finite dimensional $K$-vector space. In particular, $\Lambda$ is a graded $\Lambda$-module. Given a graded module, it has a projective cover in the category of graded modules and degree zero maps, and, its kernel is again a graded module in our sense. Note also, that as a graded algebra, $\Lambda$ is generated in degrees 0 and 1 .

Proposition 1.5. Let $M_{\Lambda}$ be a graded $\Lambda$-module. Then, the projective resolution $(\mathcal{P}, \delta)$ of Definition 1.1 can be chosen to be a graded resolution of $M_{\Lambda}$.

Proof. Since $M_{\Lambda}$ is graded, we now take $\amalg f_{i}^{0} R \rightarrow M \rightarrow 0$ as a degree 0 homomorphism with the $f_{i}^{0}$ 's homogeneous elements of $R$ in the appropriate degrees. We have a sequence of $R$-modules

$$
0 \rightarrow \Omega_{R}^{1} M \rightarrow \amalg f_{i}^{0} R \rightarrow M \rightarrow 0
$$

which is exact in gr $R$. Since $\Omega_{R}^{1}(M)$ is a graded submodule, we may take $\amalg f_{i}^{1 *} R=$ $\Omega_{\Lambda}^{1}(M)$ with the elements $f_{i}^{1 *}$ right uniform homogeneous elements of $\amalg_{i} f_{i}^{0} R$. Since $I$ is a homogeneous ideal of $R$, it follows that $I$ is also a homogeneous right ideal of $R$. Thus $\amalg f_{0} R$ is also a graded submodule of $\amalg f^{0} R$. Therefore, we have that $\amalg f^{2 *} R=\left(\amalg f^{1} R\right) \cap\left(\amalg f^{0} I\right)$ is also a graded submodule of $\amalg f^{0} R$. We inductively 
construct a chain of graded $R$-submodules of $\amalg f^{0} R$ :

$$
\cdots \subset \amalg f^{n} R \subset \amalg f^{n-1} R \subset \cdots \subset \amalg f^{0} R .
$$

The result follows by taking quotients modulo $I$.

\section{Minimality}

In this section we give an example showing that the projective resolution constructed in the previous section need not to be minimal when $I$ is an admissible ideal. However, when $I$ is an admissible ideal, we prove that the elements $\left\{f^{n}\right\}$ can be chosen such that the resolution is minimal. We also compare this resolution with the Bongartz-Butler-Gruenberg resolution in [Bo].

We start with an example showing that the resolution constructed in Definition 1.1 need not be minimal.

Example 2.1. Let $R$ be the path algebra of the following quiver:

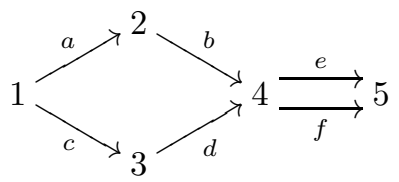

and let $I$ be the ideal of $R$ generated by $a b-c d$, bf and $d e$. Let $\Lambda=R / I$, and let $S_{1}$ be the simple $\Lambda$-module corresponding to the vertex $v_{1}$. The ideal $I$ is a 7 -dimensional vector space with a basis given by the elements $a b-c d$, abe-cde, $a b f-c d f, a b f, c d e, b f$ and $d e$. We construct now a projective resolution of $S_{1}$ over $\Lambda$ using the resolution described in Definition 1.1 .

We can take $f^{0}=v_{1}$, so we have $0 \rightarrow \Omega_{R}^{1} S_{1} \rightarrow v_{1} R \rightarrow S_{1} \rightarrow 0$ and we can decompose $\Omega_{R}^{1} S_{1}=f_{1}^{1} R \amalg f_{2}^{1} R$, where $f_{1}^{1}=a$ and $f_{2}^{1}=c$. Next we note that $\amalg f^{2 *} R=(a R \amalg b R) \cap v_{1} I=v_{1} I$, and a $K$-basis of $v_{1} I$ is the set $\{a b-c d, a b e-$ $c d e, a b f-c d f, a b f, c d e\}$. We decompose $v_{1} I$ as

$$
v_{1} I=(a b-c d) R \amalg a b f R \amalg c d e R .
$$

But $a b f$ and $c d e$ are in $\amalg f^{1} I=a I \amalg c I$. So $f^{2}=a b-c d$ and we have $f^{2} R=$ $(a b-c d) R$. Now we compute $f^{3 *}$. We have that $\amalg f^{3 *} R=(a b-c d) R \cap(a I \amalg c I)$, and it easy to check that $\amalg f^{3 *} R=(0)$, so that we obtain the following projective $\Lambda$-resolution of $S_{1}$, which turns out to be minimal:

$$
0 \rightarrow(a b-c d) R /(a b-c d) I \rightarrow a R / a I \amalg c R / c I \rightarrow v_{1} R / v_{1} I \rightarrow S_{1} \rightarrow 0 .
$$

We remark that we could have decomposed $v_{1} I$ also in the following way: $v_{1} I=$ $c d f R \amalg(a b-c d) R \amalg c d e R$ and $c d e R$ is contained in $a I \amalg c I$, but $c d f$ and $(a b-c d)$ are not in $a I \amalg c I$. So we can write $f_{1}^{2}=c d f$ and $f_{2}^{2}=a b-c d$. We continue and get $\amalg f^{3 *} R=(c d f R \amalg(a b-c d) R) \cap(a I \amalg c I)=a b f R$. Finally, we get the following $\Lambda$-projective resolution of $S_{1}$, which is clearly not minimal:

$$
\begin{aligned}
0 \rightarrow a b f R / a b f I & \rightarrow c d f R / c d f R \amalg(a b-c d) R /(a b-c d) I \\
& \rightarrow a R / a I \amalg c R / c I \rightarrow v_{1} R / v_{1} I \rightarrow S_{1} \rightarrow 0 .
\end{aligned}
$$

The next result shows, as in the above example, that we can always choose the elements $\left\{f^{n}\right\}$ in such a way that we obtain a minimal projective resolution of a finitely generated $\Lambda$-module when $I$ is an admissible ideal. 
Assume now that $I$ is an admissible ideal, hence $\Lambda$ is finite dimensional. Choose $\left\{f_{i}^{0}\right\}$ such that $\amalg_{i} f_{i}^{0} R / \amalg_{i} f_{i}^{0} I$ is a projective cover of $M_{\Lambda}$. We have the following.

Theorem 2.2. In the resolution $(\mathcal{P}, \delta)=\left(\amalg f_{i}^{n} R / f_{i}^{n} I,\left(\bar{h}^{n-1, n}\right)\right)$ the elements $\left\{f_{j}^{n}\right\}$ can be chosen in such a way that, for each $n$, no proper $K$-linear combination of a subset of $\left\{f_{j}^{n}\right\}$ is in $\amalg f^{n-1} I+\amalg f^{n *} J$.

Moreover, there is a decomposition

$$
\amalg f^{n *} R=\left(\amalg f_{i}^{n} R\right) \amalg\left(\amalg f_{i}^{n^{\prime}} R\right),
$$

where the elements $f_{i}^{n^{\prime}}$ can be chosen to be in $\amalg f^{n-1} I$.

Proof. For each $n \geq 2$ we have the decomposition

$$
\amalg f^{n *} R=\left(\amalg f^{n-1} R\right) \cap\left(\amalg f^{n-2} I\right)
$$

Step 1: We show first that we can adjust the decomposition (1) to obtain a decomposition of the type

$$
\amalg f^{n *} R=\left(f_{1}^{n} R \amalg \ldots \amalg f_{t}^{n} R\right) \amalg\left(f_{t+1}^{n^{\prime}} R \amalg \cdots \amalg f_{k}^{n^{\prime}} R\right),
$$

where each $f_{j}^{n^{\prime}}$ is in $\amalg f^{n-1} I+\amalg f^{n *} J$, and, no proper $K$-linear combinations of a subset of $\left\{f_{i}^{n}\right\}$ is in $\amalg f^{n-1} I+\amalg f^{n *} J$. To prove this claim, start with the decomposition (11). If $x=\alpha_{1} f_{1}^{n}+\cdots+\alpha_{s} f_{s}^{n}$ is a $K$-linear combination, where, say $\alpha_{1} \neq 0$, then we have $f_{1}^{n} R \amalg \ldots \amalg f_{t}^{n} R=x R \amalg f_{2}^{n} R \amalg \ldots \amalg f_{t}^{n} R$. Thus, if $x$ is in $\amalg f^{n-1} I+\amalg f^{n *} J$, we adjust our initial decomposition to the decomposition

$$
\amalg f^{n *} R=\left(f_{2}^{n} R \amalg \ldots \amalg f_{t}^{n} R\right) \amalg\left(x R \amalg f_{t+1}^{n^{\prime}} R \amalg \ldots \amalg f_{k}^{n^{\prime}} R\right),
$$

$x$ thus becoming one of the $f^{n^{\prime}}$ 's. The element $x$ may be assumed to be right uniform. We continue this process and the claim is proved.

Step 2: By the first step, we may assume that we have a decomposition of the type

$$
\amalg f^{n *} R=\left(f_{1}^{n} R \amalg \ldots \amalg f_{t}^{n} R\right) \amalg\left(f_{t+1}^{n^{\prime}} R \amalg \ldots \amalg f_{k}^{n^{\prime}} R\right),
$$

where each of the $f^{n^{\prime}}$ 's is in $\amalg f^{n-1} I+\amalg f^{n *} J$. We show now that we can further adjust this decomposition in such a way that each $f_{i}^{n^{\prime}}$ is in fact in $\amalg_{i} f_{i}^{n-1} I$.

Let $y=f_{j}^{n^{\prime}}$ be such that $f_{j}^{n^{\prime}}$ is not in $\amalg f^{n-1} I$. We can write $y=a^{\prime}+b^{\prime}$ where $a^{\prime}$ is in $\amalg f^{n-1} I$ and $b^{\prime}$ is in $\amalg f^{n *} J$. But $\amalg f^{n-1} I$ is contained in $\amalg f^{n *} R=$ $\left(\amalg f^{n-1} R\right) \cap\left(\amalg f^{n-2} I\right)$, so we can write $a^{\prime}=y a-q$ and $b^{\prime}=y b+q$ for some $a$ in $R, b$ in $J$ and some $q$ in $\amalg_{f^{n *} \neq y} f^{n *} R$.

We get $y=y(a+b)$ and, since $y$ is right uniform with terminus $w$, we have $a+b=w$, so $a=w-b$. Let $z=(w+b)\left(w+b^{2}\right)\left(w+b^{4}\right) \cdots\left(w+b^{2^{n}}\right)$. We multiply $a^{\prime}=y a-q$ in $\amalg f^{n-1} I$ by $z$ on the right and we obtain $y\left(w-b^{2^{n+1}}\right)-q z$ in $\amalg f^{n-1} I$ or $y-y b^{2^{n+1}}-q z$ in $\amalg f^{n-1} I$. Since $I$ is admissible, for large enough $n, b^{2^{n+1}}$ is in $I$, so $y b^{2^{n+1}}$ is in $y I \subset \amalg f^{n-1} I$, so $y-q z$ is in $\amalg f^{n-1} I$.

We claim now that $(y-q z) R \amalg\left(\amalg_{f^{n *} \neq y} f^{n *} R\right)=\amalg f^{n *} R$. To show this we first observe that $\amalg f^{n *} R=(y-q z) R+\left(\amalg_{f^{n *} \neq y} f^{n *} R\right)$. It is obvious that the sum is direct.

In this way it is clear that we can adjust our decomposition, and that we may assume that each of the $f_{i}^{n^{\prime}}$ is in fact in $\amalg f^{n-1} I$. The case where $n=1$ is similar. 
We now give the analogous result for the graded (not necessarily noetherian) case.

Theorem 2.3. Assume $\Lambda$ is length graded and let $M$ be a graded $\Lambda$-module. Then the resolution $(\mathcal{P}, \delta)=\left(\amalg_{i} f_{i}^{n} R / \amalg_{i} f_{i}^{n} I,\left(\bar{h}^{n-1, n}\right)\right)$ can be chosen to be a graded resolution in such a way that for each $n$, the $f_{i}^{n *}$ 's are homogeneous elements (and hence the $f_{i}^{n}$ 's), with no proper $K$-linear combination of a subset of $\left\{f_{j}^{n}\right\}$ is in $\amalg f^{n-1} I+\amalg f^{n *} J$. Moreover, there is a decomposition

$$
\amalg f^{n *} R=\left(\amalg f^{n} R\right) \amalg\left(\amalg f^{n^{\prime}} R\right)
$$

where the elements $f^{n^{\prime}}$ can be chosen to be homogeneous elements in $\amalg f^{n} I$.

Proof. By Proposition 1.5 we begin with a graded resolution of $M$. For each $n \geq 2$, we have a decomposition

$$
\amalg f^{n *} R=\left(\amalg f^{n-1} R\right) \cap\left(\amalg f^{n-2} I\right)
$$

with the $f^{n *}$ 's homogeneous.

Step 1: We show first that we may adjust the decomposition (3), to obtain a decomposition of the type

$$
\amalg f^{n *} R=\left(\amalg f^{n} R\right) \amalg\left(\amalg f^{n^{\prime}} R\right)
$$

where each $f^{n^{\prime}}$ is a homogeneous element in $\amalg f^{n-1} R+\amalg f^{n *} J$, and, no proper $K$-linear combination of a subset of $\left\{f^{n}\right\}$ is in $\amalg f^{n-1} I+\amalg f^{n *} J$. For each degree, there are only a finite number of $f^{n *}$ 's in that degree, since each homogeneous component of $M$ and $\Lambda$ is finite dimensional. Fixing a degree, we obtain

$$
\amalg f^{n *} R=\left(f_{1}^{n} R \amalg \cdots \amalg f_{t}^{n} R\right) \amalg\left(f_{t+1}^{n^{\prime}} \amalg \cdots \amalg f_{k}^{n^{\prime}} R\right)
$$

and we proceed, degree by degree, as in the proof of step 1 of Theorem 2.2,

Step 2: By the first step, we may assume that we have a collection of decompositions of the type

$$
\amalg f^{n *} R=\left(f_{1}^{n} R \amalg \cdots \amalg f_{t}^{n} R\right) \amalg\left(f_{t+1}^{n^{\prime}} \amalg \cdots \amalg f_{k}^{n^{\prime}} R\right),
$$

where all $f^{n *}, f_{i}^{n}$ and $f_{j}^{n^{\prime}}$ are homogeneous in the same degree, and, where each of the $f_{j}^{n^{\prime}}$ 's is in $\amalg f^{n-1} I+\amalg f^{n *} J$. We show now that we can adjust these decompositions in such a way that, each $f_{j}^{n^{\prime}}$ is in fact in $\amalg f^{n-1} I$. Let $y=f_{j}^{n^{\prime}}$ be such that $y$ is not in $\amalg f^{n-1} I$, and of degree $k$. We can write $y=a^{\prime}+b^{\prime}$ where $a^{\prime}$ is in $\amalg f^{n-1} I$, $b^{\prime}$ is in $\amalg f^{n *} J$, and both are homogeneous of degree $k$. Since $\amalg f^{n-1} I$ is contained in $\amalg f^{n *} R$, we can write $a^{\prime}=y a-q$ for some $q$ in $\amalg_{f^{n *} \neq y} f^{n *} R$, homogeneous of degree $s$. Note that $a$ must be a homogeneous element of $R_{0}=(K Q)_{0}$. By right uniformity $y a=y$. Thus $y-q$ is in $\amalg f^{n-1} I$ and we also have

$$
(y-q) R \amalg\left(\amalg_{f^{n *} \neq y} f^{n *} R\right)=\amalg f^{n *} R .
$$

This completes the proof.

We can now show that the adjusted resolution is minimal.

Theorem 2.4. Let $M$ be a $\Lambda$-module and let $(\mathcal{P})=\left(\amalg f^{n} R / \amalg f^{n} I,\left(\bar{h}^{n-1, n}\right)\right)$ be the projective resolution of $M$ as in Theorem 1.2 , where the representatives $\left\{f^{n}\right\}$ are chosen in such a way, that for each $n$, no proper $K$-linear combination of a subset of $\left\{f^{n}\right\}$ lies in $\amalg f^{n-1} I+\amalg f^{n *} J$. Then, the resolution $(\mathcal{P})$ is minimal. 
Proof. It is enough to show that for each $n$, the entries $h^{n-1, n}$ are in $J$, where $f^{n}=\sum f^{n-1} h^{n-1, n}$. We prove this for each $n$, the case $n=1$ being obvious.

Assume that for some $n>1$ we have a representative $f_{j}^{n}$ written as $f_{j}^{n}=$ $f_{1}^{n-1} h_{1}+\cdots+f_{t}^{n-1} h_{t}$, where not all $h_{i}$ are in $J$. Using the fact that the $f^{n-1}$ 's are right uniform elements of $R$, we get an expression

$$
f_{j}^{n}=f_{1}^{n-1} \alpha_{1}+\cdots+f_{t}^{n-1} \alpha_{t}+f_{1}^{n-1} r_{1}+\cdots+f_{t}^{n-1} r_{t}
$$

where $\alpha_{i}$ is in $K$ and $r_{i}$ is in $J$ for $i=1, \ldots, t$ (not all necessarily nonzero). Let $x=$ $\sum_{i=1}^{t} f_{i}^{n-1} \alpha_{i}$. Then we can write $x$ as $x=f_{j}^{n}-\sum_{i=1}^{t} f_{i}^{n-1} r_{i}$ in $\amalg f^{n-2} I+\amalg f^{n-1} J$, which is a contradiction to the choice of the elements $\left\{f^{n-1}\right\}$.

A projective resolution of $\Lambda / \underline{r}$ and the groups $D \operatorname{Ext}_{\Lambda}^{n}(\Lambda / \underline{\mathrm{r}}, \Lambda / \underline{\mathrm{r}})$ are given in [Bo], where $D=\operatorname{Hom}_{K}(, K)$ is the usual duality. We now give some information on the elements $f^{n}$ 's, which enables us to give a connection between the BongartzButler-Gruenberg resolution in [Bo] and our resolution.

Proposition 2.5. Let $M$ be a $\Lambda$-module and, for each $n \geq 0$, choose $\left\{f^{n}\right\}$ in such a way that the resolution $\left(\amalg f^{n} R / \amalg f^{n} I, \bar{h}\right)$ is minimal as in Theorem 2.4 .

(a) Let $n \geq 1$ and write $f^{n}=f_{1}^{n-1} r_{1}+\cdots+f_{t}^{n-1} r_{t}$ with $r_{i}$ in $R$. Then, the elements $f^{n}$ can be chosen (adjusted) in such a way that each of the elements $r_{1}, \ldots, r_{t}$ are in $J \backslash I$.

(b) The elements $f^{n+1}$ are in $\left(\amalg f^{n} J\right) \cap\left(\amalg f^{n-1} I\right)$ and

$$
\left(\amalg f^{n} J\right) \cap\left(\amalg f^{n-1} I\right) \subset \begin{cases}\left(\amalg f^{0} J I^{m}\right) \cap\left(\amalg f^{0} I^{m} J\right) & \text { if } n=2 m, \\ \left(\amalg f^{0} I^{m+1}\right) \cap\left(\amalg f^{0} J I^{m} J\right) & \text { if } n=2 m+1 .\end{cases}
$$

(c) $\Omega_{\Lambda}^{n}(M) / \Omega_{\Lambda}^{n}(M) \underline{\mathrm{r}} \simeq \amalg f^{n} R /\left(\left(\amalg f^{n} R\right) \cap\left(\amalg f^{n-1} I\right)+\amalg f^{n} J\right)$ and

$$
\amalg f^{n-1} I+\amalg f^{n} J \subset \begin{cases}\amalg f^{0} J I^{m}+\amalg f^{0} I^{m} J & \text { if } n=2 m, \\ \amalg f^{0} I^{m+1}+\amalg f^{0} J I^{m} J & \text { if } n=2 m+1 .\end{cases}
$$

(d) $D \operatorname{Ext}_{\Lambda}^{n}(M, \Lambda / \underline{\mathrm{r}}) \simeq \amalg f^{n} R /\left(\left(\amalg f^{n} R\right) \cap\left(\amalg f^{n-1} I\right)+\amalg f^{n} J\right)$.

Proof. (a) It is clear that not all the $r_{i}$ 's can be in $I$. Write, say,

$$
f_{1}^{n}=f_{1}^{n-1} r_{1}+\cdots+f_{k}^{n-1} r_{k}+f_{k+1}^{n-1} r_{k+1}+\cdots+f_{s}^{n-1} r_{s}
$$

where $r_{1}, \ldots, r_{k}$ are not in $I$, but $r_{k+1}, \ldots, r_{s}$ are all in $I$.

Let $x_{1}=f_{1}^{n-1} r_{1}+\cdots+f_{k}^{n-1} r_{k}$. Then it is easy to check that $\amalg f_{i}^{n *} R=x_{1} R \amalg$ $\left(f_{2}^{n *} R \amalg \ldots \amalg f^{n *} R\right)$, since $x_{1}=f_{1}^{n}-u$, where $u$ is in $\amalg f^{n-1} I \subset\left(\amalg f^{n-1} R\right) \cap$ $\left(\amalg f^{n-2} I\right)=\amalg f^{n *} R$. Furthermore, $x_{1}$ is not in $\amalg f^{n-1} I+\amalg f^{n *} J$; otherwise, $f_{1}^{n}$ is. So we can replace $f_{1}^{n}$ with $x_{1}$. Continue this process. The fact that the coefficients are in $J$ follows from the minimality of the projective resolution.

(b) We have that $f^{n+1}=\sum f^{n} h^{n, n+1}$, where $h^{n, n+1}$ is in $J$, since the resolution is minimal. Therefore, $f^{n+1}$ is in $\sum f^{n} J$. Moreover,

$$
f^{n+1}=\sum f^{n} h^{n, n+1}=\sum f^{n-1} h^{n-1, n} h^{n, n+1},
$$

where the last sum is in $\amalg f^{n-1} I$, since we have a resolution over $\Lambda=R / I$. It follows immediately from this that $f^{n+1}$ is in $\left(\amalg f^{n} J\right) \cap\left(\amalg f^{n-1} I\right)$.

We saw above that $f^{i}$ is in $\amalg f^{i-2} I$ for $i \geq 2$. This implies that $f^{2 m}$ is in $\amalg f^{0} I^{m}$ and that $f^{2 m+1}$ is in $\amalg f^{1} I^{m} \subset \amalg f^{0} J I^{m}$. The last claim in (b) follows immediately from this. 
(c) and (d) The second claim of (c) follows in a similar fashion to (b). Since the projective resolution of $M$ is minimal and $\Lambda / \underline{r}$ is semisimple, it follows immediately that

$$
D \operatorname{Ext}_{\Lambda}^{n}(M, \Lambda / \underline{\mathrm{r}}) \simeq \operatorname{Tor}_{n}^{\Lambda}(M, \Lambda / \underline{\mathrm{r}}) \simeq \amalg f^{n} R / \amalg f^{n} J .
$$

Furthermore, the minimality of the resolution, and the above imply that $\amalg f^{n} J=$ $\amalg f^{n} J+\left(\amalg f^{n} R\right) \cap\left(\amalg f^{n-1} I\right)$. The result follows.

It is shown in Bo that

$$
D \operatorname{Ext}_{\Lambda}^{n}(\Lambda / \underline{\mathrm{r}}, \Lambda / \underline{\mathrm{r}})= \begin{cases}\left(I^{m} \cap J I^{m-1} J\right) /\left(J I^{m}+I^{m} J\right) & \text { for } n=2 m \geq 2, \\ \left(J I^{m} \cap I^{m} J\right) /\left(I^{m+1}+J I^{m} J\right) & \text { for } n=2 m+1 \geq 1\end{cases}
$$

We see that the formulas for $D \operatorname{Ext}_{\Lambda}^{n}(\Lambda / \underline{\mathrm{r}}, \Lambda / \underline{\mathrm{r}})$ and for $D \operatorname{Ext}_{\Lambda}^{n}(M, \Lambda / \underline{\mathrm{r}})$ in Proposition 2.5 are very similar, when $M$ is any finitely generated $\Lambda$-module.

\section{EXT-ALGEBRAS}

Let $\Lambda=R / I$ where $R=K Q$ and $I \subseteq J^{2}$ is an admissible ideal of $R$, or a length homogeneous ideal. Let $\Lambda_{0}=R / J$. Note that $\Lambda_{0}$ is a semisimple $\Lambda$ module. We recall that the Ext-algebra of $\Lambda$ is the graded $K$-algebra $E(\Lambda)=$ $\amalg_{n \geq 0} \operatorname{Ext}_{\Lambda}^{n}\left(\Lambda_{0}, \Lambda_{0}\right)$ with the obvious addition and with multiplication given by the Yoneda product. Given a right $\Lambda$-module $M$, we have a graded left $E(\Lambda)$-module $E(M)=\amalg_{n \geq 0} \operatorname{Ext}_{\Lambda}^{n}\left(M, \Lambda_{0}\right)$. In this section we show how to use the minimal projective resolutions introduced in section 2 , to effectively describe the $E(\Lambda)$ action on $E(M)$ (and thus, if $M=\Lambda_{0}$, the multiplicative structure of $E(\Lambda)$ itself), in an algorithmic way, by working at the level of the path algebra $K Q$.

We start by recalling the following interpretation of the Yoneda product, which will be used in this section. First, we observe that if $M$ is a finitely generated $\Lambda$-module, in the admissible case, or $M$ is graded in the length homogeneous case, we may consider a minimal $\Lambda$-resolution of $M$ :

$$
\cdots \rightarrow P_{n} \stackrel{\delta^{n}}{\longrightarrow} P_{n-1} \rightarrow \cdots \rightarrow P_{0} \rightarrow M \rightarrow 0 .
$$

This resolution is minimal in the sense that, for each $n>0$, we have $\operatorname{Im} \delta^{n} \subseteq P_{n-1} \underline{\mathrm{r}}$ where $\underline{r}$ is the Jacobson radical of $\Lambda$ in the case where $I$ is admissible, and $\underline{r}$ is the graded radical of $\Lambda$ in the graded case which need not be finite dimensional. Since $\operatorname{Ext}_{\Lambda}^{n}\left(M, \Lambda_{0}\right)$ is the cohomology of the complex

$$
0 \rightarrow \operatorname{Hom}_{\Lambda}\left(P_{0}, \Lambda_{0}\right) \stackrel{\delta_{*}}{\longrightarrow} \operatorname{Hom}_{\Lambda}\left(P_{1}, \Lambda_{0}\right) \stackrel{\delta_{*}}{\longrightarrow} \cdots
$$

and, since $\Lambda_{0}$ is semisimple, the boundary maps of this complex are all zero. It follows that, for each $n \geq 0$, we have $\operatorname{Ext}_{\Lambda}^{n}\left(M, \Lambda_{0}\right)=\operatorname{Hom}_{\Lambda}\left(P_{n}, \Lambda_{0}\right)$. Now let $\hat{f}$ be in $\operatorname{Ext}_{\Lambda}^{n}\left(M, \Lambda_{0}\right)$ and let $\hat{g}$ be in $\operatorname{Ext}_{\Lambda}^{m}\left(\Lambda_{0}, \Lambda_{0}\right)$. We have the following commutative 
diagram with exact rows:

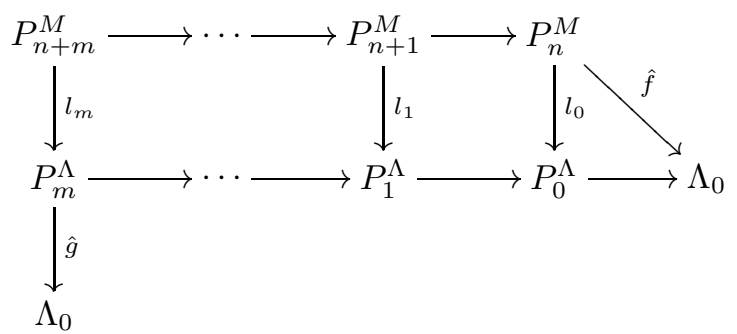

where the top row is part of a minimal projective resolution of $M$, the bottom sequence is part of a minimal projective resolution of $\Lambda_{0}$, and the vertical maps $l_{0}, l_{1}, \ldots, l_{m}$ are successive liftings (not necessarily unique) of $\hat{f}$. Then we have the following description of the $E(\Lambda)$ action on $E(M)$ :

$$
\hat{g} * \hat{f}=\text { the composition } \hat{g} \circ l_{m} .
$$

It is well known that this action is well defined. We also remark that, since $P_{n}^{M}=$ $\amalg_{i}\left(f_{i}^{n} R / f_{i}^{n} I\right)$, we have a dual basis of $\operatorname{Ext}_{\Lambda}^{n}\left(M, \Lambda_{0}\right)=\operatorname{Hom}_{\Lambda}\left(P_{n}^{M}, \Lambda_{0}\right)$ consisting of the maps $\left\{\hat{f}_{i}^{n}\right\}$ defined in the obvious way. In order to describe this action at the level of the path algebra $K Q$, we construct a commutative diagram of $R$-modules where, the first and the third rows are minimal projective $\Lambda$-resolutions of $\Omega_{\Lambda}^{n} M$ and, $\Lambda_{0}$ respectively, and the back face of the parallelepiped is the front face modulo the ideal $I$ :

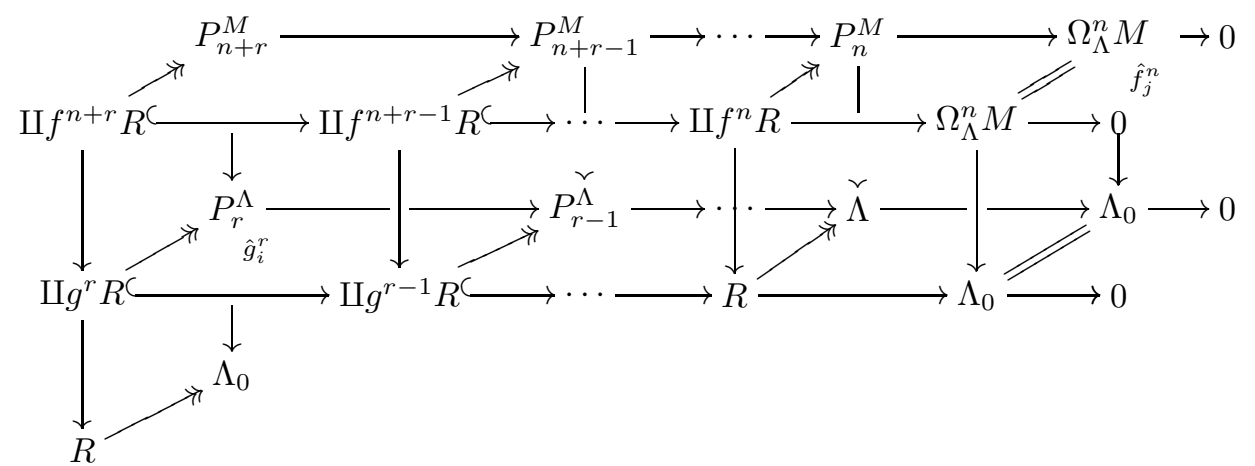

(Note that in order not to complicate our already rather complicated notation, we denote by $\hat{g}_{i}^{r}$ the map $\amalg g_{i}^{r} R \rightarrow R$ as well as its image modulo $I, \amalg \bar{g}_{i}^{r} \Lambda \rightarrow \Lambda_{0}$.)

Notation. Keeping the notation of section 2, let $\left(\amalg f_{i}^{n} R / \amalg f_{i}^{n} I,\left(\bar{h}^{n-1, n}\right)\right)$ be a minimal projective resolution of $M$ over $\Lambda$, and let $\left(\amalg g_{i}^{n} R / \amalg g_{i}^{n} I,\left(\bar{k}^{n-1, n}\right)\right)$ be a minimal projective resolution of $\Lambda_{0}$ over $\Lambda$. Recall that the entries of the matrices $\left(\bar{h}^{n-1, n}\right)$ and $\left(\bar{k}^{n-1, n}\right)$ are given by the expressions

$$
f_{i}^{n}=\sum_{j} f_{j}^{n-1} h_{j, i}^{n-1, n} \text { and } g_{i}^{n}=\sum_{j} g_{j}^{n-1} k_{j, i}^{n-1, n} .
$$

Recall also that for each $n \geq 2$ we have

$$
\left(\amalg f^{n-1} R\right) \cap\left(\amalg f^{n-2} I\right)=\left(\amalg f^{n} R\right) \amalg\left(\amalg f^{n^{\prime}} R\right)
$$


where each $f^{n^{\prime}}$ is in $\amalg f^{n-1} I$. We have similar statements and notation involving the $g$ 's, and, note that there are no $g^{0^{\prime}}$ 's and $g^{1^{\prime}}$ 's appearing.

We have the following lemma which is valid over an arbitrary ring $S$.

Lemma 3.1. Let $A, B, C$ and $D$ be $S$-modules satisfying $B \subseteq C$ and $(A+C) \cap D=$ (0). Then we have

$$
(A \amalg B \amalg D) \cap(C \amalg D) \subseteq(A \cap C) \amalg B \amalg D .
$$

Proof. We first observe that we have the inclusion $(A \amalg B \amalg C) \cap(C \amalg D) \subseteq$ $[(A \amalg B) \cap C] \amalg D$ since, if $x=a+b+d=c+d^{\prime}$, then $a+b-c=d^{\prime}-d$ is in $D$, since $b$ is also in $C$, we get that $d=d^{\prime}$ and $a+b=c$. Therefore, $x$ is in $[(A \amalg B) \cap C]+D$. But $D \cap(A+C)=(0)$ and $B \subseteq C$ so this sum is direct. The lemma follows now immediately since $B \subseteq C$ implies the well-known equality $(A \amalg B) \cap C=(A \cap C) \amalg B$.

The following rather technical proposition is crucial to our description.

Proposition 3.2. (a) For each $n \geq 0$, the matrix $\left(h^{n, n+1}\right)$ has entries in $\amalg g^{1} R$.

(b) For each $r \geq 2$ and $n \geq 0$, the product of matrices $\left(h^{n, n+1}\right) \cdots\left(h^{n+r-1, n+r}\right)$ has entries in $\left(\amalg g^{r} R\right) \amalg\left(\amalg_{2 \leq i \leq r} g^{i^{\prime}} R\right)$.

Note that for simplicity we are using the following notation: the matrix $\left(h^{n, n+1}\right)$ actually denotes the matrix $\left(h_{j, i}^{n, n+1}\right)$ where the entries $h_{j, i}^{n, n+1}$ are obtained by expanding the elements of the form $f^{n+1}$ in terms of the elements of the form $f^{n}$.

Proof. (a) From the minimality of the projective resolution of $M$ it follows that each entry of the form $h_{j, i}^{n, n+1}$ is in $J$, so we can factor out the initial arrows, (note that the family $\left\{g^{1}\right\}$ is the set of arrows of $Q$ !), and we are done.

(b) We proceed in 2 steps.

Step 1: We show first that the sum is direct. Recall that, for each $p>0$ we have $\left(\amalg g^{p} R\right) \amalg\left(\amalg g^{p^{\prime}} R\right) \subseteq \amalg g^{p-1} R$. Assume that the sum $\left(\amalg g^{r} R\right)+\left(\amalg_{k \leq i \leq r} g^{i^{\prime}} R\right)$ is direct. But this sum is a submodule of $\amalg g^{k-1} R$. Therefore its intersection with $\amalg g^{k-1^{\prime}} R$ is zero. This proves that the sum $\left(\amalg g^{r} R\right)+\left(\amalg_{k-1 \leq i \leq r} g^{i^{\prime}} R\right)$ is also direct, so it follows by induction that our sum is direct.

Step 2: We have the following:

$$
\begin{aligned}
& \left(h^{n, n+1}\right) \cdots\left(h^{n+r-1, n+r}\right) \\
& =\left[\left(h^{n, n+1}\right) \cdots\left(h^{n+r-3, n+r-2}\right)\right] \cdot\left[\left(h^{n+r-2, n+r-1}\right)\left(h^{n+r-1, n+r}\right)\right] .
\end{aligned}
$$

By induction, the product of the first $r-2$ matrices has entries in $\left(\amalg g^{r-2} R\right) \amalg$ $\left(\amalg_{2 \leq i \leq r-2} g^{i^{\prime}} R\right)$, and it is clear that the product of the last two matrices has entries in $I$, hence the entire product has entries in $\left(\amalg g^{r-2} I\right) \amalg\left(\amalg_{2 \leq i \leq r-2} g^{i^{\prime}} R\right)$. But, by looking at the product of the first $r-1$ matrices, we see that the entire product has entries also in $\left(\amalg g^{r-1} R\right) \amalg\left(\amalg_{2 \leq i \leq r-1} g^{i^{\prime}} R\right)$. Hence, the entries of the product lie in the intersection

$$
\left[\left(\amalg g^{r-1} R\right) \amalg\left(\amalg_{2 \leq i \leq r-1} g^{i^{\prime}} R\right)\right] \cap\left[\left(\amalg g^{r-2} I\right) \amalg\left(\amalg_{2 \leq i \leq r-2} g^{i^{\prime}} R\right)\right] .
$$

We now claim that we have the following inclusion:

$$
\begin{gathered}
{\left[\left(\amalg g^{r-1} R\right) \amalg\left(\amalg_{2 \leq i \leq r-1} g^{i^{\prime}} R\right)\right] \cap\left[\left(\amalg g^{r-2} I\right) \amalg\left(\amalg_{2 \leq i \leq r-2} g^{i^{\prime}} R\right)\right]} \\
\left.\quad \subseteq\left[\left(\amalg g^{r-1} R\right) \cap\left(\amalg g^{r-2} I\right)\right] \amalg\left(\amalg_{2 \leq i \leq r-1} g^{i^{\prime}} R\right)\right] .
\end{gathered}
$$


Note that, since $\left.\left(\amalg g^{r-1} R\right) \cap\left(\amalg g^{r-2} I\right)\right]=\left(\amalg g^{r} R\right) \amalg\left(\amalg g^{r^{\prime}} R\right)$, the claim will indeed imply that the entries of our product of matrices are in the required sum. To prove this claim, let $A=\amalg g^{r-1} R, B=\amalg g^{r-1^{\prime}} R, C=\amalg g^{r-2} I$, and $D=\amalg_{2 \leq i \leq r-2} g^{i^{\prime}} R$. With this notation we must prove that we have an inclusion:

$$
(A \amalg B \amalg D) \cap(C \amalg D) \subseteq(A \cap C) \amalg B \amalg D .
$$

This is precisely the statement of Lemma 3.1 once one shows that $B \subseteq C$ and that $(A+C) \cap D=(0)$. It is clear that $B \subseteq C$. We also have that $A$ and $C$ are included in $\amalg g^{r-2} R$ and $D \cap\left(\amalg g^{r-2} R\right)=(0)$; hence $D \cap(A+C)=(0)$. The proof is now complete.

Definition 3.3. It follows from the previous proposition that each entry of the matrix product $\left(h^{n, n+1}\right) \cdots\left(h^{n+r-1, n+r}\right)$ is an element of the form $\sum g^{r} l^{r, n, n+r}+$ $\sum_{2 \leq i \leq r} g^{i^{\prime}} s^{i, n, n+r}$. Let $\left(l^{r, n, n+r}\right)$ be the corresponding matrix. (Note that the number of rows of this matrix is the number of elements in the family $\left\{g^{r}\right\}$, and that the number of columns is the number of elements in the family $\left\{f^{n+r}\right\}$.)

Proposition 3.4. For each $n \geq 0$ and $r \geq 0$, the following diagram of $R$-module commutes modulo I:

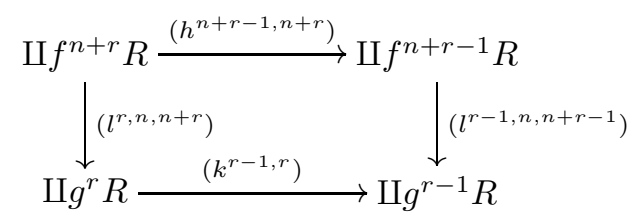

Proof. We shall use a Sweedler-type notation in order to avoid multiple indices. Note that by Proposition 3.2 we have that the entries of the product of $r$ consecutive $\left(h^{n+i, n+i+1}\right)$-matrices have the form $X=\sum g^{r} l^{r, n, n+r}+\sum_{2 \leq i \leq r} g^{i^{\prime}} s^{i, n, n+r}$. We have the following:

$$
\begin{aligned}
X= & \sum g^{r} l^{r, n, n+r}+\sum g^{r^{\prime}} s^{r, n, n+r}+\sum_{2 \leq i \leq r-1} g^{i^{\prime}} s^{i, n, n+r} \\
= & \sum\left(\sum g^{r-1} k^{r-1, r}\right) l^{r, n, n+r} \\
& +\sum\left(\sum g^{r-1} a^{r-1, r}\right) s^{r, n, n+r}+\sum_{2 \leq i \leq r-1} g^{i^{\prime}} s^{i, n, n+r} \\
= & \sum g^{r-1}\left(\sum k^{r-1, r} l^{r, n, n+r}+a^{r-1, r} s^{r, n, n+r}\right)+\sum_{2 \leq i \leq r-1} g^{i^{\prime}} s^{i, n, n+r},
\end{aligned}
$$

where each $a^{r-1, r}$ is in the ideal $I$.

On the other hand, by expressing the matrix product as

$$
\left[\left(h^{n, n+1}\right) \cdots\left(h^{n+r-2, n+r-1}\right)\right] \cdot\left(h^{n+r-1, n+r}\right),
$$

we see that each entry of this product is an entry of the product

$$
\left(\sum g^{r-1} l^{r-1, n, n+r-1}+\sum_{2 \leq i \leq r-1} g^{i^{\prime}} s^{i, n, n+r-1}\right) \cdot\left(h^{n+r-1, n+r}\right) .
$$

Therefore, it has the form

$$
\sum g^{r-1}\left(\sum l^{r-1, n, n+r-1} h^{n+r-1, n+r}\right)+\sum_{2 \leq i \leq r-1} g^{i^{\prime}}\left(\sum s^{i, n, n+r-1} h^{n+r-1, n+r}\right) .
$$


From the uniqueness of the direct sum decomposition we obtain

$$
\begin{aligned}
& \sum g^{r-1}\left(\sum l^{r-1, n, n+r-l} h^{n+r-1, n+r}\right) \\
& =\sum g^{r-1}\left(\sum k^{r-1, r} l^{r, n, n+r}+a^{r-1, r} s^{r, n, n+r}\right)
\end{aligned}
$$

where we recall that each $a^{r-1, r}$ is in $I$. It is immediate that the above equality implies the proposition.

We are ready to prove the main result of this section.

Theorem 3.5. Let $\hat{f}_{j}^{n}$ be in $\operatorname{Ext}_{\Lambda}^{n}\left(M, \Lambda_{0}\right)$ and let $\hat{g}_{i}^{r}$ be in $\operatorname{Ext}_{\Lambda}^{r}\left(\Lambda_{0}, \Lambda_{0}\right)$ be two basis elements. Then, the element $\hat{g}_{i}^{r} * \hat{f}_{j}^{n}$ of $\operatorname{Ext}_{\Lambda}^{n+r}\left(M, \Lambda_{0}\right)$ is given by

$$
\hat{g}_{i}^{r} * \hat{f}_{j}^{n}=\sum_{t} \tilde{l}_{i, j, t}^{r, n, n+r} \hat{f}_{t}^{n+r}
$$

where $\tilde{l}$ is the image in $\Lambda / \underline{\mathrm{r}}$ of the element $l$ of $R$.

Proof. We infer from the previous proposition that for each $n \geq 0$, and $r>1$, we have a commutative diagram of $\Lambda$-modules:

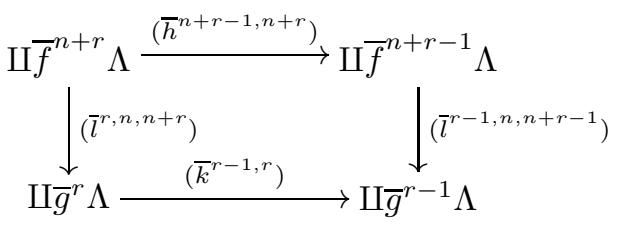

This shows, as promised, that we have constructed a diagram (5) as in the beginning of this section, and, that the product $\hat{g}_{i}^{r} * \hat{f}_{j}^{n}$ is in fact the composition $\hat{g}_{i}^{r} \circ\left(\bar{l}^{r, n, n+r}\right)$. The theorem now follows.

\section{The NO LOOP CONJECTURE}

This section is devoted to applying the minimal projective resolution found in section 2 to the no loop conjecture. In particular, we show a special case of this conjecture.

Let $K, Q$, and $R$ be as above, and let $I$ be an admissible ideal in $R$. As before, denote $R / I$ by $\Lambda$. The no loop conjecture says the following. If $S$ is a simple $\Lambda$ module such that $\operatorname{Ext}_{\Lambda}^{1}(S, S) \neq(0)$, then $\operatorname{pd}_{\Lambda} S=\infty$. In [I] , Igusa proved that the global dimension of $\Lambda$ must be infinite, whenever there exists a simple $\Lambda$-module $S$ with $\operatorname{Ext}_{\Lambda}^{1}(S, S) \neq(0)$. (See also $[\mathrm{L}$.)

First we prove a small technical lemma.

Lemma 4.1. Suppose $a$ is an arrow in $Q$, where $a: v \rightarrow v$ for some vertex $v$ in $Q$. Let $S$ be the simple $\Lambda$-module corresponding to this vertex, and assume that the minimal resolution of $S$ has been constructed finding the elements $f^{n}, f^{n^{\prime}}$ and $h^{n-1, n}$. The residue class of $a^{n}$ in $v I / v(J I+I J)$ is nonzero if and only if

$$
a^{n}=\sum_{i} f_{i}^{2} r_{i}+\sum_{i} f_{i}^{2^{\prime}} s_{i}
$$

where the coefficients $r_{i}$ and $s_{i}$ are $R$, and where some $r_{i}$ is not in $J$. 
Proof. Assume that $a^{n}=\sum_{i} f_{i}^{2} r_{i}+\sum_{i} f_{i}^{2^{\prime}} s_{i}$ for $r_{i}$ and $s_{i}$ in $R$, where one of the $r_{i}$ 's is not in $J$. Assume that the residue class of $a^{n}$ in $v I / v(J I+I J)$ is zero. Since each $f_{i}^{2^{\prime}}$ is in $\amalg f^{1} I$, it is in $v J I$. Hence the set $\left\{f_{i}^{2}\right\}$ is linearly dependent in $v I / v(J I+I J)$. This is a contradiction to the choice of $f_{i}^{2}$ (Theorem 2.4), so that the residue class of $a^{n}$ in $v I / v(J I+I J)$ is nonzero.

Conversely, assume that $a^{n}=\sum_{i} f_{i}^{2} r_{i}+\sum_{i} f_{i}^{2^{\prime}} s_{i}$ for some $r_{i}$ and $s_{i}$ in $R$, where all $r_{i}$ are in $J$. Then clearly the residue class of $a^{n}$ in $v I / v(J I+I J)$ is zero.

Now we show that if $S$ is a simple $\Lambda$-module corresponding to a vertex with a loop $a$ where $a^{n}$ is in $I$ and $a^{n}$ has a nonzero residue class in $v I / v(J I+I J)$, then $\operatorname{pd}_{\Lambda} S=\infty$.

Proposition 4.2. Suppose $a$ is an arrow in $Q$, where $a: v \rightarrow v$ for some vertex $v$ in $Q$. Let $S$ be the simple $\Lambda$-module corresponding to this vertex, and assume that $a^{n}$ is in $I$ for some $n \geq 2$ and that the residue class of $a^{n}$ in $v I / v(J I+I J)$ is nonzero. Then $\operatorname{Ext}_{\Lambda}^{i}(S, S) \neq(0)$ for all $i \geq 1$. In particular, $\operatorname{pd}_{\Lambda} S=\infty$.

Proof. Note that the hypothesis implies that $a^{n-1}$ is not in $I$. It is well-known that $\operatorname{Ext}_{\Lambda}^{i}(S, S) \neq(0)$ for $i=1,2$.

By the above lemma, we have that $a^{n}=\sum_{i} f_{i}^{2} r_{i}^{2}+\sum_{i} f_{i}^{2^{\prime}} s_{i}^{2}$ for some $r_{i}^{2}$ and $s_{i}^{2}$ in $R$, where some $r_{i}^{2}$ is not in $J$. Let $z_{2}=\sum_{i} f_{i}^{2^{\prime}} s_{i}^{2}$. Then $b_{2}=a^{n}-z_{2}$ is in $\amalg f^{2} R$. We want to show that $b_{2} a$ is in $\amalg f^{2} R \cap \amalg f^{1} I$. Since $a^{n}$ is in $I$ and $z_{2}$ is in $\amalg f^{2^{\prime}} R$, the element $z_{2}$ is in $\amalg f^{1} I$ and, therefore $b_{2} a$ is in $\amalg f^{2} R \cap \amalg f^{1} I$.

Assume that $b_{2} a$ is in $\amalg f^{2} I$. Then there exist $u_{i}$ in $I$ such that $\sum_{i} f_{i}^{2} r_{i}^{2} a=$ $\sum_{i} f_{i}^{2} u_{i}$. Hence, $r_{i}^{2} a$ is in $I$ for all $i$. There exists an $i_{0}$ such that $r_{i_{0}}^{2}=c v+h$ for $c$ in $K^{*}$ and $h$ in $J$. Then

$$
c^{-1}\left(v+\left(c^{-1} h\right)^{2^{m}}\right) \cdots\left(v+\left(c^{-1} h\right)^{2}\right)\left(v-\left(c^{-1} h\right)\right)(c v+h) a=a-\left(c^{-1} h\right)^{2^{m+1}}
$$

is in $I$. Since $I$ is admissible and $h$ is in $J$, for some large $m$, we get that $a$ is in $I$. This is a contradiction, which shows that $b_{2} a$ is not in $\amalg f^{2} I$ and, $\operatorname{Ext}_{\Lambda}^{3}(S, \Lambda / \underline{r}) \neq$ (0).

By the above, we can write $b_{2} a$ as

$$
b_{2} a=\sum_{i} f_{i}^{3} r_{i}^{3}+\sum_{i} f_{i}^{3^{\prime}} s_{i}^{3}
$$

for some elements $r_{i}^{3}$ and $s_{i}^{3}$ in $R$. Expanding the two sides in the elements $f^{2}$, we get

$$
\sum_{i} f_{i}^{2} r_{i}^{2} a=\sum_{t, i} f_{t}^{2} h_{t, i}^{2,3} r_{i}^{3}+\sum_{t, i} f_{t}^{2} \alpha_{t, i}^{3} s_{i}^{3}
$$

where each $\alpha_{i}^{3}$ is in $I$. This implies that

$$
r_{t}^{2} a=\sum_{i} h_{t, i}^{2,3} r_{i}^{3}+\sum_{i} \alpha_{t, i}^{3} s_{i}^{3} .
$$

There exists some $t$ such that $r_{t}^{2} a$ is not in $J^{2}$. If $r_{i}^{3}$ is in $J$ for all $i$, then the right hand side is in $J^{2}$. Therefore, we infer that not all $r_{i}^{3}$ are in $J$. Since $b_{2} a$ ends in $v$, there are also some $f_{i}^{3}$ such that $f_{i}^{3} v \neq 0$. Hence, $\operatorname{Ext}_{\Lambda}^{3}(S, S) \neq(0)$. Let $z_{3}=\sum_{i} f^{3^{\prime}} s_{i}^{3}$ and $b_{3}=b_{2} a-z_{3}$.

Assume now that we have shown that

(a) $b_{2 m+1}=b_{2 m} a-z_{2 m+1}=\sum_{i} f_{i}^{2 m+1} r_{i}^{2 m+1}$, where not all $r_{i}^{2 m+1}$ are in $J$,

(b) $b_{2 m}=\sum_{i} f_{i}^{2 m} r_{i}^{2 m}$, where not all $r_{i}^{2 m}$ are in $J$, 
(c) $z_{2 m+1}$ is in $\amalg f^{2 m+1^{\prime}} R$.

We want to show that $b_{2 m+1} a^{n-1}$ represents some nonzero element in $\operatorname{Ext}_{\Lambda}^{2 m+2}(S, S)$.

The element $b_{2 m+1} a^{n-1}$ is clearly in $\amalg f^{2 m+1} R$. Since $b_{2 m}$ is in $\amalg f^{2 m} R$ and $z_{2 m+1}$ is in $\amalg f^{2 m} I$, we have that $b_{2 m+1} a^{n-1}=b_{2 m} a^{n}+z_{2 m+1} a^{n-1}$ is in $\amalg f^{2 m} I$. Therefore, $b_{2 m+1} a^{n-1}$ is in $\amalg f^{2 m+1} R \cap \amalg f^{2 m} I$.

Assume that $b_{2 m+1} a^{n-1}$ is in $\amalg f^{2 m+1} I$, that is, $b_{2 m+1} a^{n-1}=\sum f_{i}^{2 m+1} u_{i}^{2 m+1}$, where $u_{i}^{2 m+1}$ is in $I$ for all $i$. Expanding the elements in terms of the elements $f^{2 m}$ we get

$$
\begin{aligned}
\sum_{i} f_{i}^{2 m} r_{i}^{2 m} a^{n} & =\sum_{i} f_{i}^{2 m+1} u_{i}^{2 m+1}+z_{2 m+1} a^{n-1} \\
& =\sum_{t} f_{t}^{2 m} h_{t, i}^{2 m, 2 m+1} u_{i}^{2 m+1} \sum_{t} f_{t}^{2 m} \beta_{t}^{2 m+1} a^{n-1}
\end{aligned}
$$

where $\beta_{t}^{2 m+1}$ is in $I$ for all $t$. For each $t$, we have that

$$
r_{t}^{2 m} a^{n}=\sum_{i} h_{t, i}^{2 m, 2 m+1} u_{i}^{2 m+1}+\beta_{t}^{2 m+1} a^{n-1} .
$$

The right hand side is in $J I+I J$ for all $t$. For some $t$, the element $r_{t}^{2 m}$ is not in $J$. Using that $I$ is an admissible ideal and similar arguments as before, we conclude that $a^{n}$ is in $J I+I J$. This is a contradiction, and therefore $b_{2 m+1} a^{n-1}$ is not in $\amalg f^{2 m+1} I$ and $\operatorname{Ext}_{\Lambda}^{2 m+2}(S, \Lambda / \underline{\mathrm{r}}) \neq(0)$.

By the above, $b_{2 m+1} a^{n-1}$ has a representation of the form

$$
b_{2 m+1} a^{n-1}=\sum_{i} f_{i}^{2 m+2} r_{i}^{2 m+2}+\sum_{i} f_{i}^{2 m+2^{\prime}} s_{i}^{2 m+2} .
$$

Expanding in terms of the elements $f^{2 m}$, we get

$$
\begin{aligned}
\sum_{i} f_{i}^{2 m} r_{i}^{2 m} a^{n}= & \sum_{i} f_{i}^{2 m+2} r_{i}^{2 m+2}+\sum_{i} f_{i}^{2 m+2^{\prime}} s_{i}^{2 m+2}+z_{2 m+1} a^{n-1} \\
= & \sum f_{t}^{2 m} h^{2 m, 2 m+1} h^{2 m+1,2 m+2} r_{i}^{2 m+2} \\
& \quad+\sum f_{l}^{2 m+1} \alpha_{l, i}^{2 m+2} s_{i}^{2 m+2}+z_{2 m+1} a^{n-1} \\
= & \sum f_{t}^{2 m} h^{2 m, 2 m+1} h^{2 m+1,2 m+2} r_{i}^{2 m+2} \\
& \quad+\sum f_{t}^{2 m} h^{2 m, 2 m+1} \alpha_{l, i}^{2 m+2} s_{i}^{2 m+2}+z_{2 m+1} a^{n-1}
\end{aligned}
$$

for some $\alpha_{l, i}^{2 m+2}$ in $I$. We have that $z_{2 m+1}$ is in $\amalg f^{2 m} I$, so that, if $r_{i}^{2 m+2}$ is in $J$ for all $i$, then $r_{i}^{2 m} a^{n}$ is in $J I+I J$ for all $i$. Since there exists $r_{i_{0}}^{2 m}$ which is not in $J$, we show as before that $a^{n}$ is in $J I+I J$. This is a contradiction, and consequently, not all $r_{i}^{2 m+2}$ are in $J$, and $\operatorname{Ext}_{\Lambda}^{2 m+2}(S, S) \neq(0)$. Let $z_{2 m+2}=\sum_{i} f^{2 m+2^{\prime}} s_{i}^{2 m+2}$ and $b_{2 m+2}=b_{2 m+1} a^{n-1}-z_{2 m+2}$. We can now move on to the following step.

Assume now that we have shown that

(a) $b_{2 m}=b_{2 m-1} a^{n-1}-z_{2 m}=\sum_{i} f_{i}^{2 m} r_{i}^{2 m}$, where not all $r_{i}^{2 m}$ are in $J$,

(b) $b_{2 m-1}=\sum_{i} f_{i}^{2 m-1} r_{i}^{2 m-1}$, where not all $r_{i}^{2 m-1}$ are in $J$,

(c) $z_{2 m}$ is in $\amalg f^{2 m^{\prime}} R$.

We want to show that $b_{2 m} a$ represents some nonzero element in $\operatorname{Ext}_{\Lambda}^{2 m+1}(S, S)$.

The element $b_{2 m} a$ is clearly in $\amalg f^{2 m} R$. Since $b_{2 m-1}$ is in $\amalg f^{2 m-1} R, z_{2 m}$ is in $\amalg f^{2 m-1} I$ and $b_{2 m} a=b_{2 m-1} a^{n}-z_{2 m} a$, we have that $b_{2 m} a$ is in $\amalg f^{2 m} R \cap \amalg f^{2 m-1} I$. 
Assume that $b_{2 m} a$ is in $\amalg f^{2 m} I$. Then $b_{2 m} a=\sum_{i} f_{i}^{2 m} r_{i}^{2 m} a=\sum_{i} f_{i}^{2 m} u_{i}^{2 m}$ with $u_{i}^{2 m}$ in $I$. Therefore, $r_{i}^{2 m} a$ is in $I$ for all $i$, and, as before, this would give the contradiction that $a$ is in $I$. Thus $b_{2 m} a$ is not in $\amalg f^{2 m} I$ and $\operatorname{Ext}_{\Lambda}^{2 m+1}(S, \Lambda / \underline{\mathrm{r}}) \neq(0)$.

By the above, the element $b_{2 m} a$ has a representation of the form

$$
b_{2 m} a=\sum_{i} f_{i}^{2 m+1} r_{i}^{2 m+1}+\sum_{i} f^{2 m+1^{\prime}} s_{i}^{2 m+1} .
$$

Expanding in terms of the elements $f^{2 m}$, we get

$$
\begin{aligned}
\sum_{i} f_{i}^{2 m} r_{i}^{2 m} a & =\sum f_{i}^{2 m+1} r_{i}^{2 m+1}+\sum_{i} f_{i}^{2 m+1^{\prime}} s_{i}^{2 m+1} \\
& =\sum_{t} f_{t}^{2 m} h_{t, i}^{2 m, 2 m+1} r_{i}^{2 m+1}+\sum_{t} f_{t}^{2 m} \alpha_{t, i}^{2 m+1} s_{i}^{2 m+1}
\end{aligned}
$$

where $\alpha_{t, i}^{2 m+1}$ is in $I$. If all $r_{i}^{2 m+1}$ are in $J$, then $r_{i}^{2 m} a$ is in $J^{2}$ for all $i$. As before, this is a contradiction, and therefore, there exists some $r_{i}^{2 m+1}$ not in $J$. Using the same arguments as before, it follows that $\operatorname{Ext}_{\Lambda}^{2 m+1}(S, S) \neq(0)$. Combining what we have shown so far, the proof of the proposition is complete.

We point out that it follows from our proof of Proposition 4.2 that the residue class of $a^{r n}$ is nonzero in $J I^{r-1} J \cap I^{r} /\left(J I^{r}+I^{r} J\right)$ (which is isomorphic to $\operatorname{Tor}_{2 r}^{\Lambda}(\Lambda / \underline{\mathrm{r}}, \Lambda / \underline{\mathrm{r}})$ by $\left.[\mathrm{BO}]\right)$, and that the residue class of $a^{r n+1}$ is nonzero in $J I^{r} \cap I^{r} J /\left(J I^{r} J+I^{r+1}\right)$ (which in turn is isomorphic to $\left.\operatorname{Tor}_{2 r+1}^{\Lambda}(\Lambda / \underline{\mathrm{r}}, \Lambda / \underline{\mathrm{r}})\right)$.

However, when $\operatorname{Ext}_{\Lambda}^{1}(S, S) \neq(0)$, then $\operatorname{Ext}_{\Lambda}^{i}(S, S)$ is not in general nonzero for all $i \geq 1$. The following example was pointed out to us by Dieter Happel [H2].

Let $Q$ be the quiver given by

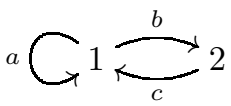

Let $I$ be the ideal in $K Q$ generated by $\left(a^{2}-b c, c a b, c b\right)$. Denote by $S$ the simple $\Lambda$ module corresponding to the vertex 1 . As with all simple modules corresponding to a vertex with a loop, $\operatorname{Ext}_{\Lambda}^{i}(S, S) \neq(0)$ for $i=1,2$. However, $\operatorname{Ext}_{\Lambda}^{3}(S, S)=(0)$, and, since $\Omega_{\Lambda}^{4}(S)=S$, then $\operatorname{Ext}_{\Lambda}^{i}(S, S)=(0)$ if $i \equiv 3 \bmod 4$, and nonzero otherwise for all $i \geq 1$.

\section{EXAMPles}

Let $Q$ be a finite quiver and let $I=\left\langle\rho_{1}, \ldots, \rho_{n}\right\rangle$ be an admissible ideal of $K Q$, where $\left\{\rho_{1}, \ldots, \rho_{n}\right\}$ is a minimal set of generators of the two-sided ideal $I$. Assume that for each $i=1, \ldots, n$, the element $\rho_{i}$ is a linear combination of paths in $Q$ with coefficients +1 or -1 . Let $\Lambda=K Q / I$ be the corresponding finite dimensional algebra. We know that if $\Lambda$ is a monomial algebra, then the global dimension of $\Lambda$ (more generally, the projective dimension of each simple $\Lambda$-module) is independent of the characteristic of $K$ (see GHZ]). If $\Lambda$ is the incidence algebra of a partially ordered set, the global dimension of $\Lambda$, although always finite, can vary with the characteristic of $K$, unless $\operatorname{gldim} \Lambda \leq 2$ in which case it is again characteristic independent (see [C, IZ]). In this section, we give examples which show that the global dimension can fluctuate rather wildly according to the characteristic of the field. The proofs of these examples can be easily done using the minimal projective resolution constructed in the second section. We start, however, by showing that, 
if the global dimension is two in one characteristic, then it cannot be infinite in another. More specifically, we prove the following.

Theorem 5.1. Let $Q$ be a finite quiver and let $E$ and $F$ be two fields. Let $\rho_{1}, \ldots, \rho_{n}$ be linear combinations of paths in $Q$, where the coefficients are \pm 1 , and let $I_{E}$ ( $I_{F}$ respectively) denote the two-sided ideal of $E Q$ (FQ respectively) generated by the elements $\rho_{1}, \ldots, \rho_{n}$. Assume that $I_{E}$ and $I_{F}$ are both admissible ideals, and, that gldim $E Q / I_{E} \leq 2$. Then gldim $F Q / I_{F}<\infty$.

We will prove the theorem by induction on the number of vertices of $Q$, the case where we have only one vertex being obvious. We need a few preliminary results.

Lemma 5.2. Let $\Lambda$ be an artin algebra and let $S_{\Lambda}$ be a simple $\Lambda$-module such that $\operatorname{id}_{\Lambda} S \leq 1$. Let e be the primitive idempotent corresponding to $S$. Then,

(i) $\operatorname{gldim}(1-e) \Lambda(1-e) \leq \operatorname{gldim} \Lambda$,

(ii) if $\operatorname{gldim} \Lambda=\infty$, then $\operatorname{gldim}(1-e) \Lambda(1-e)=\infty$. (Compare with Fu, Proposition 2.5].)

Proof. Since $\operatorname{id}_{\Lambda} S_{\Lambda} \leq 1$, we have $\operatorname{pd}_{\Lambda \text { op }} D S \leq 1$, where $D$ is the usual duality, and we can use [Z to infer (i). Assume now that $\operatorname{gldim} \Lambda=\infty$. Then there is a $\Lambda$-module $M_{\Lambda}$ such that $\operatorname{pd} M_{\Lambda}=\infty$. Let $N=\Omega_{\Lambda}^{2} M$-the second syzygy of $M$. By [J], since id $S \leq 1$, we have that, if

$$
\mathcal{P}: \cdots P_{n} \rightarrow \cdots \rightarrow P_{0} \rightarrow N_{\Lambda} \rightarrow 0
$$

is a minimal projective resolution of $N$, then no $P_{i}$ has a summand isomorphic to $e \Lambda$. Thus, $\operatorname{Hom}_{\Lambda}((1-e) \Lambda, \mathcal{P})$ is a minimal projective resolution of the $(1-e) \Lambda(1-e)$ module $\operatorname{Hom}_{\Lambda}((1-e) \Lambda, N)$. Hence, gldim $(1-e) \Lambda(1-e)=\infty$.

The next result is well-known.

Lemma 5.3. Let $\Lambda=K Q / I$ be a finite dimensional quotient of the path algebra $K Q$ by an admissible ideal $I$. Let $\rho_{1}, \ldots, \rho_{n}$ be a minimal set of generators of $I$ in the sense that $I$ cannot be generated by a proper subset of $\left\{\rho_{1}, \ldots, \rho_{n}\right\}$. For a vertex $v$ of $Q$ the following are equivalent:

(i) $\operatorname{id} S_{v} \leq 1$.

(ii) $\rho_{i} v=0$ for each $i=1, \ldots, n$.

Definition 5.4. Let $Q$ be a finite quiver and let $I$ be an admissible ideal of $K Q$. Let $\rho_{1}, \ldots, \rho_{n}$ be a minimal set of generators of $I$ and assume that $\rho_{i} v=0$ for each $i$. It is clear that there are no loops at the vertex $v$ since, otherwise, we would have that $\operatorname{Ext}_{K Q / I}^{2}\left(S_{v}, S_{v}\right) \neq(0)$, contradicting the fact that the injective dimension of $S_{v}$ is less or equal to 1 . We construct a new quiver $Q^{*}$ as follows: the vertices of $Q^{*}$ are all the vertices of $Q$ different from $v$. The arrows of $Q^{*}$ are obtained from the arrows of $Q$ in the following way: each arrow of $Q$ whose origin and terminus are both different from $v$, is also an arrow of $Q^{*}$, and, for each path $a b$ in $Q$ :

$$
\stackrel{a}{\rightarrow} \underset{v}{\bullet} \stackrel{b}{\rightarrow} \underset{w}{\bullet}
$$

such that $a b$ is not in $I$, we form a "new" arrow $C(a, b)$ in $Q^{*}$

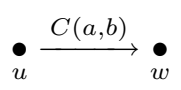


The corresponding two-sided ideal $I^{*}$ of $K Q^{*}$ is defined in the following manner: if $\rho_{i}$ is such that $v \rho_{i}=0$, and if $\rho_{i}$ is not a path of the form $a b$ :

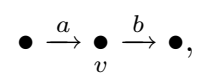

then we let $\rho_{i}^{*}$ be the relation obtained from $\rho_{i}$, by replacing each occurrence of a path of the form $\bullet \stackrel{a}{\rightarrow} \bullet \stackrel{b}{\rightarrow} \bullet$ by the arrow $C(a, b)$. Assume now that $\rho_{i}$ is a relation satisfying $v \rho_{i} \neq 0$. Let $a_{1}, \ldots, a_{k}$ denote all the arrows into the vertex $v$, and let

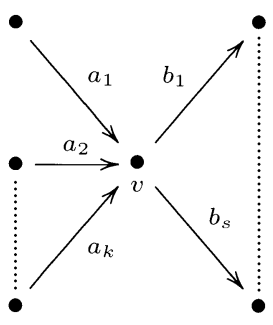

$b_{1}, \ldots, b_{s}$ denote all the arrows starting at $v$. We let $\rho_{i j}^{*}$ be the relation obtained from $a_{j} \rho_{i}$ by replacing each path $a_{j} b_{l}$ not belonging to $I$, that appears in $a_{j} \rho_{i}$, by the arrow $C\left(a_{j}, b_{l}\right)$. We now define $I^{*}$ to be the two-sided ideal of $K Q^{*}$ generated by all the elements of type $\rho^{*}$.

Proposition 5.5. There is a K-algebra isomorphism

$$
K Q^{*} / I^{*} \simeq(1-e) K Q / I(1-e),
$$

where $e$ is the primitive idempotent corresponding to the vertex $v$.

Proof. Let $\phi: K Q^{*} / I^{*} \rightarrow(1-e) K Q / I(1-e)$ be $\phi(w)=\bar{w}$ for each vertex $w$ of $Q^{*}, \phi(a)=\bar{a}$ for each arrow $a$ of $Q^{*}$ inherited from $Q$, and also $\phi(C(a, b))=\overline{a b}$ for each arrow of $Q^{*}$ of the form $C(a, b)$ (here $\bar{x}$ means the image in $K Q / I$ of the element $x$ of $K Q)$. It is clear that $\phi$ induces a $K$-algebra homomorphism which is onto, and, it is not hard to show that $\operatorname{Ker} \phi=I^{*}$.

Remark 5.6. The generating set $\left\{\rho_{i}^{*}, \rho_{i k}^{*}\right\}$ of $I^{*}$ need not be minimal. Indeed, it could happen that one of the $\rho_{i k}^{*}$ 's can be written in terms of the remaining $\rho^{*}$ 's. However, no element of the form $\rho_{i}^{*}$ can be written in terms of the remaining $\rho_{i}^{*}$ 's and $\rho_{i k}^{*}$ 's. This means that, by dropping if necessary some of the elements of the form $\rho_{i k}^{*}$, we get a minimal generating set of $I^{*}$, and, by construction the coefficients involved are again \pm 1 .

Finally, we should point out that $I^{*}$ need not be admissible, for instance, one of the minimal relations may be a sum of $C(a, b)$ and linear combinations of other paths. In this case, it is easy to see that $K Q^{*} / I^{*}$ is isomorphic to an algebra $K Q^{* *} / I^{* *}$, where $Q^{* *}$ is obtained from $Q^{*}$ by dropping the arrow $C(a, b)$, and $I^{* *}$ is obtained from $I^{*}$ in the obvious way, that is, by replacing every occurrence of $C(a, b)$ by the given linear combination.

Proof of Theorem 5.1. Any artin algebra of global dimension $n$ has at least one simple module with injective dimension equal to $n-1$ by an argument similar to the one in $[\mathrm{Z}]$. The theorem follows from the remarks above by induction on the number of nonisomorphic simple modules. 
The following examples illustrate how global dimension can be affected by the characteristic of the ground field.

Example 5.7. Let $Q$ be the following quiver:

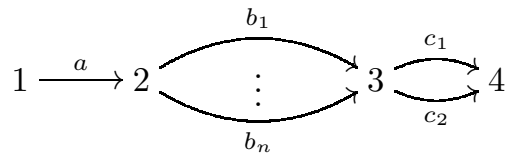

where $n \geq 2$, and, let $I=\left\langle\sum_{i=1}^{n} a b_{i}, b_{i} c_{1}+b_{n} c_{2}\right.$, for $\left.i=1, \ldots, n\right\rangle$. Let $\Lambda=K Q / I$ and let $S_{1}$ be the simple $\Lambda$-module corresponding to the vertex 1 .

We claim the following:

$$
\operatorname{pd}_{\Lambda} S_{1}= \begin{cases}3, & \text { if } \operatorname{char} k \mid n, \\ 2, & \text { if } \operatorname{char} k \nmid n\end{cases}
$$

and,

$$
\operatorname{gldim} \Lambda= \begin{cases}3, & \text { if } \operatorname{char} k \mid n, \\ 2, & \text { if } \operatorname{char} k \nmid n .\end{cases}
$$

Proof. It is easy to see that $f^{0}=v_{1}$ and $f^{1}=a$. We can write $v_{1} I \cap a R=v_{1} I=$ $\amalg_{i} f_{i}^{2 *} R$ and we can easily see that $f^{2}=\sum_{i=1}^{n} a b_{i}$, since for each $i, a b_{i} c_{1}+a b_{n} c_{2}$ is an element of $a I=f^{1} I$. Next, in computing the intersection $f^{2} R \cap f^{1} I$, we must solve the equation

$$
\sum_{i=1}^{n}\left(a b_{i} c_{1}+a b_{n} c_{2}\right) \alpha_{i}=\left(\sum_{i=1}^{n} a b_{i} c_{1}\right) \beta+\left(\sum_{i=1}^{n} a b_{i} c_{2}\right) \gamma
$$

where $\alpha_{1}, \ldots, \alpha_{n}, \beta, \gamma$ are in the field $K$. By identifying corresponding coefficients, we see that for each $i=1, \ldots, n, \alpha_{i}=\beta, \sum_{i=1}^{n} \alpha_{i}=\gamma$, and also that $\gamma=0$. This implies that $n \beta=0$, and $f^{2} R \cap f^{1} I=\left(\sum_{i=1}^{n} a b_{i} c_{1} \beta\right) R$. If char $K \nmid n$, then $\beta=0$. Thus $f^{2} R \cap f^{1} I=(0)$, and hence $\operatorname{pd} S_{1}=2$. If char $K \mid n$, then we can take $\beta=1$; we also see that $\sum_{i=1}^{n} a b_{i} c_{1}$ is not in $f^{2} I$, since $c_{1}$ is not in $I$. In this case, we have $f^{3}=\sum_{i=1}^{n} a b_{i} c_{1}$. It is easy to show that $f^{3} R \cap f^{2} I=(0)$. The remaining statements are obvious.

Example 5.8. A small adjustment of the previous example yields the following. Let $Q$ be the quiver given by

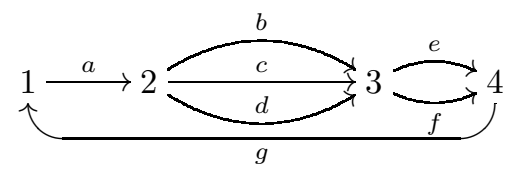

and let $I=\langle a b+a c+a d, b e+d f, c e+d f, d e+d f, e g, g a\rangle$. Let $\Lambda=K Q / I$ and let $S_{i}$ be the simple $\Lambda$-module corresponding to the vertex $i$ of $Q$. It is easy to see that $\Lambda$ is finite dimensional. An easy calculation shows that $\Lambda$ has finite global dimension if and only if the characteristic of $K \neq 3$. In characteristic different from 3 we have that $\operatorname{pd}_{\Lambda} S_{1}=2, \operatorname{pd}_{\Lambda} S_{2}=5, \operatorname{pd}_{\Lambda} S_{3}=4$ and $\operatorname{pd}_{\Lambda} S_{4}=3$. In characteristic equal to 3 all simple $\Lambda$-modules have infinite projective dimension. In fact, we have the following initial segments of the minimal projective resolutions of the simple $\Lambda$-modules. 
In characteristic 3 :

$$
\begin{gathered}
0 \rightarrow S_{1} \rightarrow P_{4} \rightarrow P_{3} \rightarrow P_{2} \stackrel{a}{\rightarrow} P_{1} \rightarrow S_{1} \rightarrow 0, \\
0 \rightarrow S_{1}^{2} \rightarrow P_{4}^{3} \rightarrow P_{3}^{3} \rightarrow P_{2} \rightarrow S_{2} \rightarrow 0, \\
0 \rightarrow S_{1} \rightarrow P_{4}^{2} \rightarrow P_{3} \rightarrow S_{3} \rightarrow 0 \\
0 \rightarrow S_{1} \rightarrow P_{4} \rightarrow S_{4} \rightarrow 0 .
\end{gathered}
$$

In characteristic different from 3 :

$$
0 \rightarrow P_{3} \stackrel{b+c+d}{\longrightarrow} P_{2} \stackrel{a}{\rightarrow} P_{1} \rightarrow S_{1} \rightarrow 0
$$

and as above $\Omega_{\Lambda}^{3}\left(S_{2}\right) \simeq S_{1}^{2}, \Omega_{\Lambda}^{2}\left(S_{3}\right) \simeq S_{1}$ and $\Omega_{\Lambda}\left(S_{4}\right) \simeq S_{1}$.

\section{APPENDIX}

The authors would like to thank M. C. R. Butler and the referee for kindly pointing out that we can also approach the construction of the projective resolutions more conceptually. One gains a notational simplicity but loses an explicit algorithmic method of constructing the resolution. We briefly sketch this approach and add some comments on how to reobtain our explicit constructions from it.

We keep the following notations from the paper. Let $R=K Q$ denote a path algebra, let $I$ be an ideal in $R$, and $\Lambda=R / I$. We let $J$ be the ideal of $R$ generated by arrows of $Q$ and let $R_{0}$ denote the $K$-span of the vertices of $Q$. Then $R_{0}$ is a semisimple $K$-algebra and $R=R_{0} \oplus J$ as $R_{0}$-modules. A right projective $R$ module is isomorphic to $U \otimes_{R_{0}} R$ where $U$ is a right $R_{0}$-module. See [BK, $\mathrm{G}$ ]. Let $M$ be a right $R / I$-module, and let $F_{0}$ be a semisimple $R_{0}$-module such that there is a surjection $F_{0} \otimes_{R_{0}} R \rightarrow M$. Let $K_{1}=\operatorname{Ker}\left(F_{0} \otimes_{R_{0}} R \rightarrow M\right)$. Then $K_{1}=F_{1} \otimes_{R_{0}} R$ for some right $R_{0}$-module $F_{1}$. We note that $F_{1}$ can be chosen to be an $R_{0}$-complement of $K_{1} J$ in $K_{1}$ which generates $K_{1}$. It should be remarked that one can always find an $R_{0}$-complement to $K_{1} J$ in $K_{1}$. Because of the special structure of projective $R$-modules, $F_{1}$ can be chosen to generate $K_{1}$. An algorithmic method for constructing $F_{1}$ can be given by constructing a minimal right uniform Gröbner basis for $K_{1}$ in $F_{0} \otimes_{R_{0}} R$. This Gröbner basis is an $R_{0}$-generating set for $F_{1}$ (see [G]). Fix $F_{1}$ with the desired properties. Then we get the beginning of a projective $R / I$-resolution of $M$.

$$
F_{1} \otimes_{R_{0}} R / F_{1} \otimes_{R_{0}} I \rightarrow F_{0} \otimes_{R_{0}} R / F_{0} \otimes_{R_{0}} I \rightarrow M \rightarrow 0 .
$$

Next, proceed inductively as follows. Let $K_{n+1}=\left(F_{n} \otimes_{R_{0}} R\right) \cap\left(F_{n-1} \otimes_{R_{0}} I\right)$ in $F_{0} \otimes_{R_{0}} R$. Let $F_{n+1}^{*}$ be an $R_{0}$-complement to $K_{n+1} J$ in $K_{n}$ which generates $K_{n+1}$. The same remarks made above about the existence and construction of $F_{1}$ apply to the existence and construction of $F_{n+1}^{*}$. Choose $F_{n+1}^{*}$ with the desired properties. Thus $K_{n+1}=F_{n+1}^{*} \otimes_{R_{0}} R$. Decompose $F_{n+1}^{*}$ as an $R_{0}$-module, $F_{n+1}^{*}=F_{n+1} \oplus F_{n+1}^{\prime}$, with $F_{n+1}^{\prime} \subseteq F_{n} \otimes_{R_{0}} I$. We get an $R / I$-projective resolution of $M$ as follows.

$$
\begin{aligned}
\cdots & \rightarrow F_{2} \otimes_{R_{0}} R / F_{2} \otimes_{R_{0}} I \rightarrow F_{1} \otimes_{R_{0}} R / F_{1} \otimes_{R_{0}} I \\
& \rightarrow F_{0} \otimes_{R_{0}} R / F_{0} \otimes_{R_{0}} I \rightarrow M \rightarrow 0 .
\end{aligned}
$$

This method yields the construction of the paper by taking the $f_{i}^{n}$ 's to be an " $R_{0}$-basis" of $F_{n}$; namely, since $F_{n}$ is a direct some of simple $R_{0}$-modules, the $f_{i}^{n}$ choose one from each summand. As remarked above, the $F_{n}$ 's and the $f_{i}^{n}$ 's in particular, can be explicitly constructed using right Gröbner basis techniques. 
In the construction in Section 1, we find the $f_{n+1}^{*}$ 's and form $F_{n+1}^{\prime}$ by taking all $f_{n+1}^{*}$ 's in $F_{n} \otimes_{R_{0}} I$. One does not need to remove all such $f_{n+1}^{*}$ 's; that is, $F_{n+1}^{\prime}$ can be chosen to be any summand of $F_{n+1}^{*}$ with the property that $F_{n+1}^{\prime} \subseteq F_{n} \otimes_{R_{0}} I$. Of course, if one is attempting to construct a minimal projective resolution, $F_{n+1}^{\prime}$ must be taken as large as possible. On the other hand, always taking $F_{n}^{\prime}=0$ yields the following well known generalization of the Gruenberg resolution mentioned in the introduction. Let $P_{0}=F_{0} \otimes_{R_{0}} R$ and $P_{1}=\operatorname{Ker}\left(P_{0} \rightarrow M\right)$ (over $R$ ). Then, the above construction yields the long exact sequence

$$
\rightarrow P_{0} I^{2} / P_{0} I^{3} \rightarrow P_{1} I / P_{1} I^{2} \rightarrow P_{0} I / P_{0} I^{2} \rightarrow P_{1} / P_{1} I \rightarrow P_{0} / P_{0} I \rightarrow M \rightarrow 0,
$$

coming from the filtration

$$
\cdots \subseteq P_{0} I^{2} \subseteq P_{1} I \subseteq P_{0} I \subseteq P_{1} \subseteq P_{0}
$$

This immediately yields the following formulae. For $m \geq 1$,

$$
\operatorname{Tor}_{2 m}^{\Lambda}\left(M, R_{0}\right)=\left(P_{1} I^{m-1} J \cap P_{0} I^{m}\right) /\left(P_{1} I^{m}+P_{0} I^{m} J\right),
$$

and for $m \geq 0$,

$$
\begin{gathered}
\operatorname{Tor}_{2 m+1}^{\Lambda}\left(M, R_{0}\right)=\left(P_{1} I^{m} \cap P_{0} I^{m} J\right) /\left(P_{1} I^{m} J+P_{0} I^{m+1}\right) . \\
\text { REFERENCES }
\end{gathered}
$$

[A] Anick, D., On the homology of associative algebras, Trans. Amer. Math. Soc. 296 (1986), no. 2,641-659. MR 87i:16046

[AG] Anick, D., Green, E. L., On the homology of quotients of path algebras, Comm. Algebra 15 (1987), no. 1-2, 309-341. MR 88c:16033

[Ba] Bardzell, M., The alternating syzygy behavior of monomial algebras, J. Algebra, 188 (1997), 1, 69-89. MR 98a:16009

[Bo] Bongartz, K., Algebras and quadratic forms, J. London Math. Soc. (2), 28 (1983) 461469. MR 85i: 16036

[BK] Butler, M. C. R., King, A., Minimal resolutions of algebras, J. Algebra, 212 (1999), 323-362. MR 2000f:16013

[C] Cibils, C., Cohomology of incidence algebras and simplicial complexes, J. Pure Appl. Algebra 56 (1989), no. 3, 221-232. MR 90d:18009

[E] Eilenberg, S., Homological dimension and syzygies, Ann. of Math., (2) 64 (1956) 328336. MR 18:558c

[ENN] Eilenberg, S., Nagao, H., Nakayama, T., On the dimension of modules and algebras IV, Dimension of residue rings of hereditary rings, Nagoya Math. J. 10 (1956) 87-95. MR 18:9e

[F] Farkas, D., The Anick resolution, J. Pure Appl. Algebra 79 (1992), no. 2, 159-168. MR 93h:16007

[FGKK] Feustel, C. D., Green, E. L., Kirkman, E., Kuzmanovich, J., Constructing projective resolutions, Comm. Algebra 21 (1993), no. 6, 1869-1887. MR 94b:16022

[Fu] Fuller, Kent R., The Cartan determinant and global dimension of Artinian rings, Azumaya algebras, actions, and modules (Bloomington, IN, 1990), 51-72, Contemp. Math., 124, Amer. Math. Soc., Providence, RI, 1992. MR 92m:16035

[G] Green, E. L., Multiplicative bases, Gröbner bases, and right Gröbner bases, to appear, J. Symbolic Comp.

[GHZ] Green, E. L., Happel, D., Zacharia, D., Projective resolutions over Artin algebras with zero relations, Illinois J. Math. 29 (1985), no. 1, 180-190. MR 86d:16031

[H1] Happel, D., Hochschild cohomology of finite dimensional algebras, Springer Lecture Notes in Mathematics, vol. 1404 (1989) 108-126. MR 91b:16012

[H2] Happel, D., Private communication.

[HZ] Zimmermann-Huisgen, B., Homological domino effect and the first finitistic dimension conjecture, Invent. Math., 108 (1992), no. 2, 369-383. MR 93i:16016

[I] Igusa, K., Notes on the no loops conjecture, J. Pure Appl. Algebra 69 (1990), no. 2, 161-176. MR 92b:16013 
[IZ] Igusa, K., Zacharia, D., On the cohomology of incidence algebras of partially ordered sets, Comm. Algebra 18 (1990), no. 3, 873-887. MR 91e:18014

[J] Jans, J. P., Some generalizations of finite projective dimension, Illinois J. Math., 5, 1961, 334-344. MR 32:1226

[L] Lenzing, H., Nilpotente Elemente in Ringen von endlicher globaler Dimension, Math. Z. 108 (1969) 313-324. MR 39:1498

[Z] Zacharia, D., On the Cartan matrix of an artin algebra of global dimension two, J. Algebra 82 (1983), no. 2, 353-357. MR 84h:16020

Department of Mathematics, Virginia Tech, Blacksburg, Virginia 24061-0123

E-mail address: green@math.vt.edu

Institutt for matematiske fag, NTNU, Lade, N-7491 Trondheim, Norway

E-mail address: oyvinso@math.ntnu.no

Department of Mathematics, Syracuse University, Syracuse, New York 13244

E-mail address: zacharia@mailbox.syr.edu 